\title{
Sleeping on the Motor Engram: The Multifaceted Nature of Sleep- Related Motor Memory Consolidation
}

Bradley R. King ${ }^{1^{*}}$, Kerstin Hoedlmoser ${ }^{2 *}$, Franziska Hirschauer ${ }^{2}$, Nina Dolfen ${ }^{1}$, Genevieve Albouy ${ }^{1}$

\author{
${ }^{1}$ Movement Control and Neuroplasticity Research Group, Kinesiology Department, KU Leuven, Leuven, \\ Belgium \\ ${ }^{2}$ Laboratory for Sleep, Cognition and Consciousness Research, Centre for Cognitive Neuroscience, \\ University of Salzburg, Salzburg, Austria \\ *Equal contribution
}

\section{Corresponding author:}

Genevieve ALBOUY

Movement Control and Neuroplasticity Research Group

Kinesiology Department - KU Leuven

Tervuurse Vest 101 - Box 1501

3001 Leuven

BELGIUM

Tel: +3216374691

Fax: + 3216329197

Email: genevieve.albouy@kuleuven.be

Web: http://faber.kuleuven.be/english/research/dep2/mcn/control/people

Running header: Sleep and Motor Memory Consolidation

Keywords: Sleep; Motor Learning; Memory Consolidation; Spindles; Slow Waves; Striatum; Hippocampus 


\section{Table of Contents}

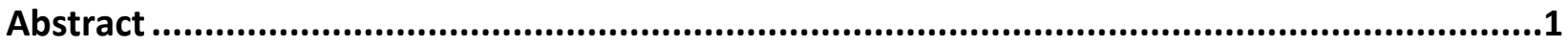

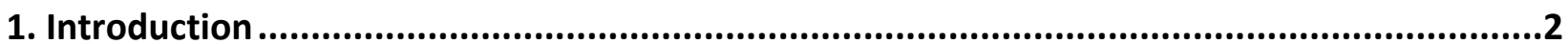

2. Influence of sleep on the behavioral correlates of motor memory consolidation..................3

2.1. Does sleep enhance performance as compared to wakefulness? ............................................ 3

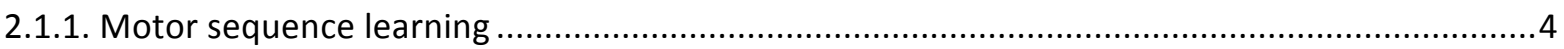

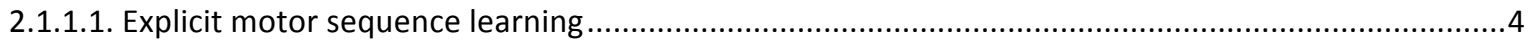

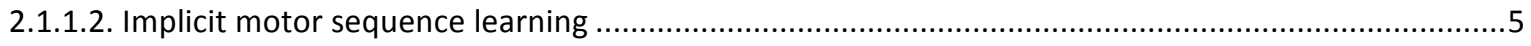

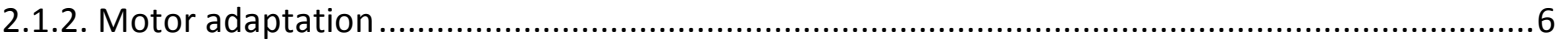

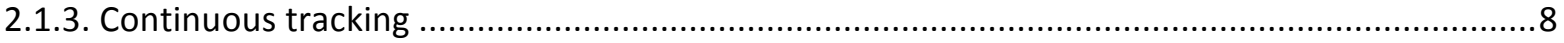

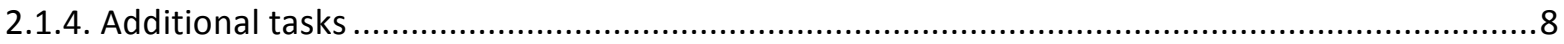

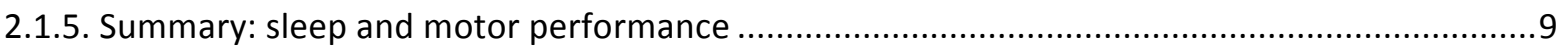

2.2. Characteristics of sleep-dependent motor memory consolidation ....................................... 9

2.2.1. Is there a temporal window during which sleep should occur to enhance consolidation? ........9

2.2.2. Does sleep help protect a learned skill from competing material? .........................................11

2.2.3. Does post-learning sleep facilitate or hinder subsequent performance on a related skill?......12

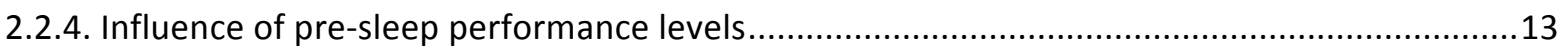

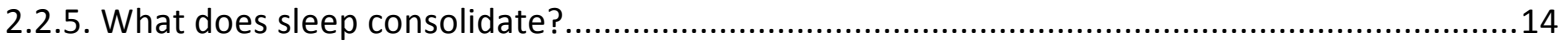

2.2.5.1. Consolidation of sequential information ................................................................................ 14

2.2.5.2. Consolidation and extraction of explicit knowledge .....................................................................15

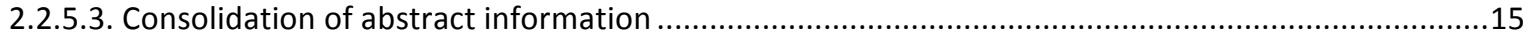

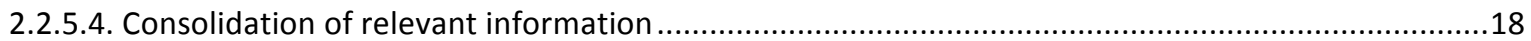

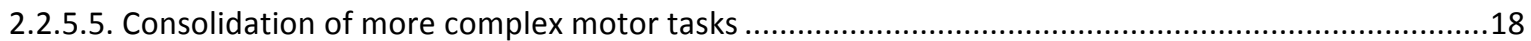

2.2.6. Summary: characteristics of sleep-dependent motor memory consolidation .........................19

3. Influence of sleep on the neural correlates of motor memory consolidation .....................19

3.1. Motor sequence learning ...............................................................................................................20

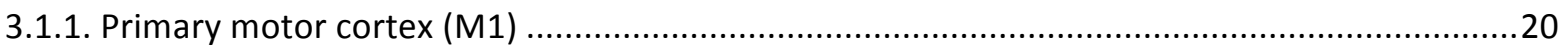

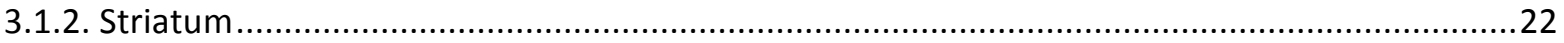




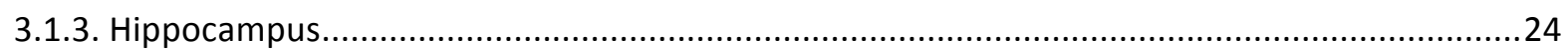

3.1.3.1. Hippocampal activity and connectivity during initial motor learning forecasts subsequent sleep-

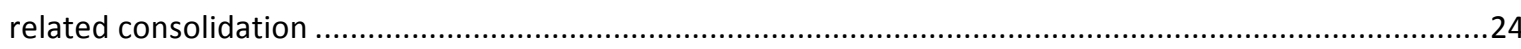

3.1.3.2. Task-related changes in hippocampal activity and connectivity after sleep...................................26

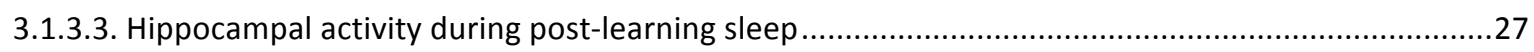

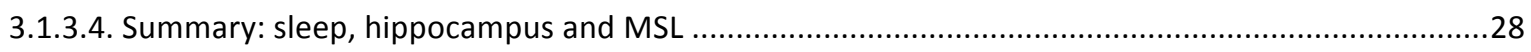

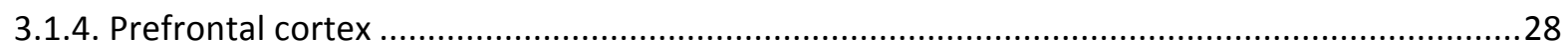

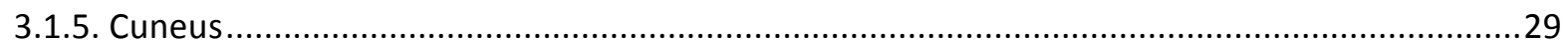

3.1.6. Premotor cortex, supplementary motor area (SMA) and pre-SMA ......................................30

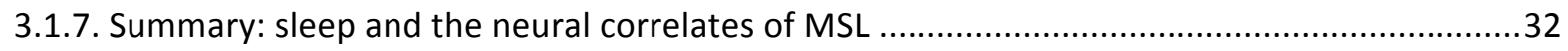

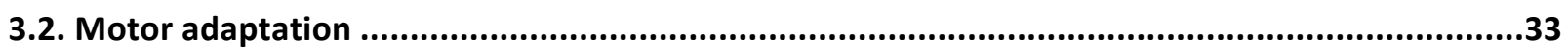

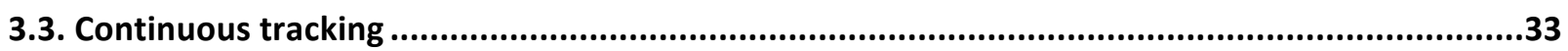

4. Sleep features involved in motor memory consolidation processes...................................34

4.1. Relationship between sleep features and motor memory consolidation ..................................34

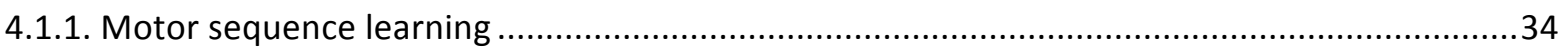

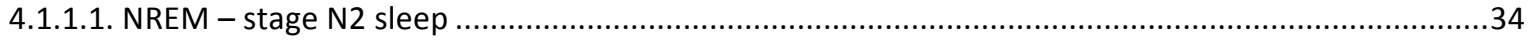

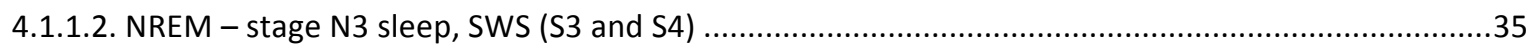

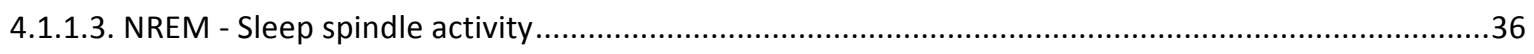

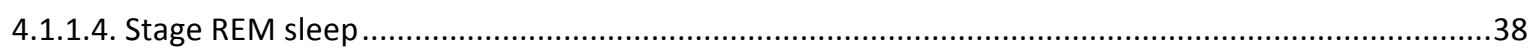

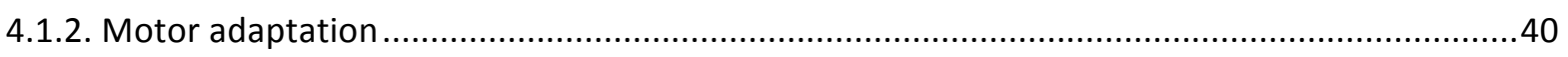

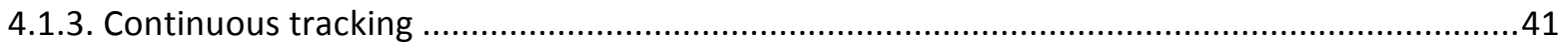

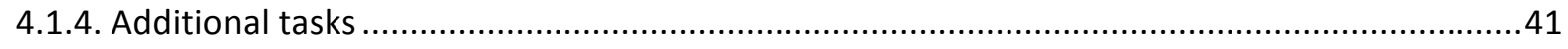

4.1.5. Summary: correlation between sleep features and motor memory consolidation ................42

4.2. Active manipulation of post-learning sleep ..........................................................43

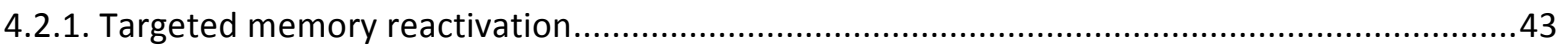

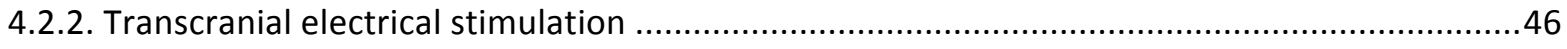

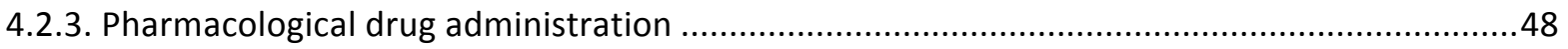

4.2.4. Summary: active manipulation of sleep and motor memory consolidation .........................49

4.3. Summary: sleep features involved in motor memory consolidation ......................................49

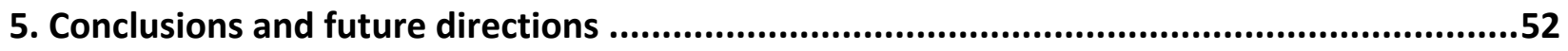




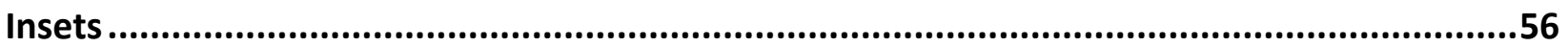

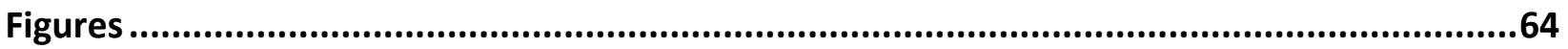

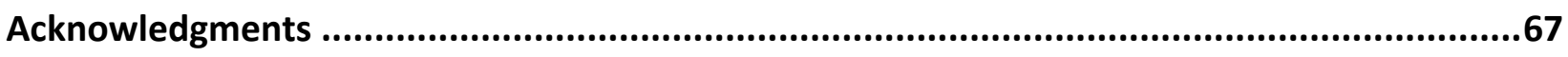

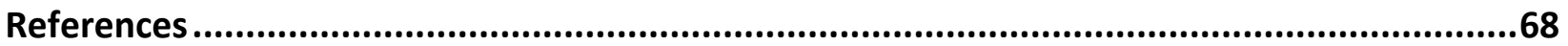




\section{Abstract}

For the past two decades, it has generally been accepted that sleep benefits motor memory consolidation processes. This notion, however, has been challenged by recent studies and thus the sleep and motor memory story is equivocal. Currently, and in contrast to the declarative memory domain, a comprehensive overview and synthesis of the effects of post-learning sleep on the behavioral and neural correlates of motor memory consolidation is not available. We therefore provide an extensive review of the literature in order to highlight that sleep-dependent motor memory consolidation depends upon multiple boundary conditions, including particular features of the motor task, the recruitment of relevant neural substrates (and the hippocampus in particular), as well as the specific architecture of the intervening sleep period (specifically, sleep spindle and slow wave activity). For our field to continue to advance, future research must consider the multifaceted nature of sleep-related motor memory consolidation. 


\section{Introduction}

Sleep has long been thought to play a beneficial role in memory consolidation, the process by which newly acquired, relatively labile memories are transformed into enhanced and more stable memory traces [e.g., see (Diekelmann et al., 2009; Frankland and Bontempi, 2005; McClelland et al., 1995) for reviews]. Although the role of sleep in memory consolidation was traditionally associated with the declarative memory system, a plethora of studies has extended these findings into the procedural, and more specifically motor, memory domain [e.g., (Fischer et al., 2002; Karni et al., 1994; Maquet, 2000; Plihal and Born, 1997; Smith and MacNeill, 1994; Walker et al., 2003)]. The influence of post-learning sleep on both the behavioral and neural correlates of motor memory consolidation is the focus of the current review.

Motor learning is known to not only take place online (i.e., during task practice) but also offline (i.e., between training sessions) in the absence of any further practice (Karni et al., 1998, 1995). This offline period is thought to offer a privileged time window for motor memory consolidation to occur. It has been approximately 20 years since the earliest studies demonstrated that post-learning sleep benefited motor memory consolidation processes (Plihal and Born, 1997; Smith and MacNeill, 1994). Since these seminal experiments, research examining the relationship between sleep and motor memory consolidation has continued to increase. This beneficial effect of post-learning sleep has been frequently replicated in subsequent experiments; however, there have also been numerous studies demonstrating no such sleep-related enhancement. Moreover, examinations into the roles of REM and NREM sleep and specific sleep features such as spindles and slow wave activity - on motor memory processing have also produced inconsistent findings. Thus, the sleep and motor memory story is currently equivocal. Accordingly, the overarching aim of this paper is to offer an exhaustive review of the effect of sleep on 
motor memory consolidation at both the behavioral and neural levels. A point of emphasis in this review is the multifaceted nature of sleep-dependent memory consolidation, as whether or not sleep affords performance benefits is heavily influenced by particular features of the motor task, the involvement of specific task-related neural substrates and post-training sleep characteristics.

Given that the effects of sleep on motor memory consolidation vary based on the motor task (see Inset 1 for a brief overview of the various motor learning paradigms), our review, when possible, will decompose the available literature based on the motor task examined. It is critical to emphasize that the vast majority of sleep and motor memory studies are based on variants of motor sequence learning paradigms and thus our review and primary conclusions reflect this lack of balance in the literature.

- INSERT INSET 1 ABOUT HERE -

\section{Influence of sleep on the behavioral correlates of motor memory consolidation}

\subsection{Does sleep enhance performance as compared to wakefulness?}

In this section, we describe how sleep and wake periods after initial training on various motor learning tasks influence performance at delayed retests (see Inset 2 for a description of the different experimental designs). We only report the studies in which the effect of sleep was statistically compared to the effect observed in a matched-control wake group. Thus, assessing whether or not a sleep group exhibited performance enhancement or maintenance from the end of the initial training session to the post-sleep retest provides little valuable information (when not compared to an appropriate wake control group), as it has recently been argued that such within-group statistical comparisons are 
confounded by non-learning and -memory related factors such as the averaging of performance across multiple blocks, fatigue, etc. [see (Adi-Japha and Karni, 2016; Pan and Rickard, 2015; Rickard and Pan, 2017) for detailed discussion of these issues]. Consequently, sleep-enhanced consolidation here refers to an advantage in performance (i.e., either enhancement or maintenance) or by a reduction in the susceptibility to interfering experiences (i.e., stabilization; see Section 2.2.2) after a period of sleep as compared to an equivalent period of wakefulness.

Please note that the purpose of this first section is simply to provide a systematic review of the effect of sleep, as compared to wakefulness, on subsequent motor behavior and thus predominantly discusses the literature in terms of whether or not a sleep-related benefit was demonstrated (i.e., a binary decomposition). This initial section is vital for readers to assess the state of the sleep and motor memory literature before proceeding to the more integrated perspectives presented in subsequent sections.

\subsubsection{Motor sequence learning}

It has been proposed that consolidation processes differ based on the awareness of the sequential material to learn (Robertson et al., 2004b); thus, we will review findings separately for explicit and implicit motor sequence learning (MSL) tasks.

\subsubsection{Explicit motor sequence learning}

A beneficial effect of sleep as compared to wakefulness on motor performance has predominantly been observed for explicit finger sequence learning tasks (Debas et al., 2010; Doyon et al., 2009b; Fischer et al., 2005, 2002; Fischer and Born, 2009; Gregory et al., 2014; Korman et al., 2003; Nettersheim et al., 2015; Nishida and Walker, 2007; Rickard et al., 2008; Robertson et al., 2004b; Schönauer et al., 2015, 2014; Siengsukon and Al-Sharman, 2011; Spencer et al., 2007; Tucker et al., 2016; Wilson et al., 2012). A similar beneficial effect of sleep was also observed for grosser explicit MSL involving sequential 
movements of the hand (Kvint et al., 2011), the arms (Kempler and Richmond, 2012; Malangré et al., 2014) as well as the whole body (Genzel et al., 2012a). This positive impact of sleep on motor sequence memory consolidation was demonstrated with a variety of tasks (i.e., finger tapping tasks, finger opposition tasks, serial reaction time tasks) and experimental designs, including diurnal and nocturnal sleep (as compared to matched wake control groups).

It should be emphasized that a smaller number of studies using finger (Albouy et al., 2013c; Backhaus et al., 2016; Cai and Rickard, 2009; Genzel et al., 2015; Landry et al., 2016; Schönauer et al., 2014) as well as

grosser [i.e., arm movements; (Gudberg et al., 2015)] explicit MSL tasks did not replicate such a beneficial effect of sleep on consolidation processes. Among the cited studies, it has been argued that this lack of an effect could be attributed to subsequent sleep episodes occurring between the sleep/wake manipulation and the retest [i.e., 2 post-sleep-deprivation recovery nights before retest; (Albouy et al., 2013c)], the time window during which sleep occurs after learning [(Cai and Rickard, 2009), but see Section 2.1.2 below], the duration of the sleep episode [i.e., 3h vs. $8 \mathrm{~h}$; (Schönauer et al., 2014)] or the intake of oral contraception (Genzel et al., 2015). Collectively, however, the majority of studies on explicit sequence learning including both fine and grosser tasks show a positive effect of sleep on consolidation processes as compared to wakefulness.

\subsubsection{Implicit motor sequence learning}

The picture is less clear regarding implicit motor sequence learning. While some studies report a beneficial effect of sleep on consolidation processes (Albouy et al., 2006; Ertelt et al., 2012; Spencer et al., 2007, 2006), others show no differential influence of sleep and wake on changes in motor sequence performance (Borragán et al., 2015; Nemeth et al., 2010; Robertson et al., 2004b; Song et al., 2007; Urbain et al., 2013). It is worth noting however, that studies showing positive effects of sleep on implicit 
motor sequence memory consolidation often used specific versions of the serial reaction time task including: (a) a greater spatial component in which the sequence of ocular movements followed a pattern of spatial locations in two dimensions (Albouy et al., 2006); (b) a contextual dimension where a sequence of color-cued movements was presented in the context of a sequence of spatial locations (Spencer et al., 2007, 2006); or, (c) a modulation of the declarative component of learning, as participants simultaneously completed a declarative word pair association and sequence learning tasks (Ertelt et al., 2012). Interestingly, these three aspects are thought to trigger sleep-related consolidation processes due to their dependency on the hippocampal system (see Sections 2.2.5 and 3.1.3). Based on these latter results, it appears that sleep-dependent consolidation can be observed for implicit motor sequence learning for specific task variants (but see Section 3.1 for additional discussion of this issue). Thus, certain features of the task, rather than solely the traditional explicit vs. implicit dissociation appear to influence the contribution of sleep in motor memory consolidation processes. The remaining sections in this review will predominantly collapse across implicit and explicit sequence learning paradigms; but, for the sake of completeness, we will note the type of task employed.

\subsubsection{Motor adaptation}

The seminal paper by Plihal and Born (1997) was one of the first to demonstrate that sleep following an initial session of a motor adaptation (MA) task (mirror tracing) increased performance in a subsequent retest. Moreover, their results indicated that this effect was specific to sleep towards the end of a night, which consists of a higher proportion of REM as compared to NREM sleep (see Inset 3). Since this first formative study, the beneficial effect of sleep on MA consolidation has been replicated on multiple occasions with different task variations and experimental designs (Albouy et al., 2013d; Backhaus and Junghanns, 2006; Huber et al., 2004; Javadi et al., 2011; Mantua et al., 2015; Schönauer et al., 2015; 
Seeck-Hirschner et al., 2010). However, and analogous to implicit motor sequence learning, sleepdependent enhancements following MA have not been consistently replicated as multiple studies reported no differences between sleep and wake control groups (Backhaus et al., 2016; Debas et al., 2010; Donchin et al., 2002; Doyon et al., 2009b; Tucker et al., 2006).

It is worth mentioning that the majority of previous research that has employed a mirror tracing task has indeed demonstrated sleep-dependent performance enhancements [(Backhaus and Junghanns, 2006; Javadi et al., 2011; Mantua et al., 2015; Plihal and Born, 1997; Schönauer et al., 2015; Seeck-Hirschner et al., 2010); but see (Tucker et al., 2006) for no effect of sleep]. As the mirror completely flips the available visual information and adults typically have ample prior experience in their daily lives with mirrors, performance may depend, at least partially, on a cognitive or explicit strategic component of the task. It is possible that the consolidation of this cognitive or declarative component underlying performance on the mirror drawing task is facilitated by post-learning sleep. However, this explanation is speculative, as no research to date has attempted to disentangle the various components of this specific task. The mechanisms underlying sleep-dependent consolidation of mirror tracing are thus not yet understood and future research should attempt to investigate why mirror tracing seems to be more sleep-dependent relative to other adaptation paradigms.

Only one study to date has examined the role of sleep on MA consolidation with a gross motor task (Hoedlmoser et al., 2015). Participants were trained to ride a bicycle with an inverted steering handle; results demonstrated that a midday nap decreased performance during slalom riding in a same-day retest. Although this result is inconsistent with the research on fine motor adaptation tasks reported above, the authors suggested that post-adaptation sleep in this context served to protect the more ecologically valid and stable motor memory of riding a normal bicycle at the expense of the newly 
acquired and less relevant memory specific for riding an inverted steering bicycle. This is consistent with research demonstrating that sleep selectively targets memories of increased relevance (Wilhelm et al., 2011 and see Section 2.2.5.4). Nonetheless, investigations into the role of sleep on MA consolidation for gross motor tasks are sparse and should be a focus of future research.

\subsubsection{Continuous tracking}

The majority of sleep studies to date that used continuous tracking tasks either did not contain a wake control group or failed to report the appropriate statistical comparisons. The remaining research is inconsistent as to whether sleep offers benefits to memory consolidation processes following motor tracking. Using sleep deprivation protocols, there is evidence that post-learning sleep enhances consolidation (Maquet et al., 2003; Smith and MacNeill, 1994). Using other experimental designs, two more recent studies did not replicate these earlier results (Borich and Kimberley, 2011; Rieth et al., 2010). There are, unfortunately, too few studies to attempt to systematically explain these discrepant results.

\subsubsection{Additional tasks}

A small subset of studies has investigated the role of sleep on motor memory consolidation using less commonly employed tasks. Gais and colleagues (2008) employed a sleep deprivation protocol and an oculomotor saccade task and revealed offline improvements only following a sleep episode. Results from Morita et al. $(2016,2012)$ demonstrated that a post-learning nap improved performance on a juggling task. And, Brawn and collaborators (2008) provided evidence that sleep facilitated offline maintenance, as compared to deterioration, in performance following learning a first-person shooter video game, representing a complex, multimodal sensorimotor skill. These additional studies build on top of the research presented earlier demonstrating that sleep can indeed facilitate memory consolidation of 
motor skills.

\subsubsection{Summary: sleep and motor performance}

The results presented above suggest that post-learning sleep tends to exert a beneficial effect on consolidation processes following explicit versions of motor sequence learning and specific task variants of implicit motor sequence learning. Altogether, it appears that sleep-dependent consolidation processes can be triggered if learning occurs under conditions known to elicit hippocampal activation during training (this issue will be discussed in more detail in Sections 2.2 .5 and 3.1.3.). It is also important to note however that results are more heterogeneous for other motor tasks.

\subsection{Characteristics of sleep-dependent motor memory consolidation}

\subsubsection{Is there a temporal window during which sleep should occur to enhance consolidation?}

The notion that sleep must occur in a critical time window following initial encoding in order to enhance memory consolidation processes was first proposed by Smith (1985). In the motor domain, this hypothesis has received particular attention for (explicit) motor sequence memory tasks. It has even been argued that the lack of beneficial effects of sleep on motor performance reported in some studies could be attributed to the fact that sleep occurred after this sensitive time window (Cai and Rickard, 2009). However, the literature does not firmly support the view that such a window exists. Indeed, the studies that have suggested this notion either were not designed to address such a question (Fischer et al., 2002) or were limited to a sample of female adolescents (Holz et al., 2012). Of note, the results in Holz et al. (2012) may also be at least partially explained by potential pre-sleep differences in performance related to time-of-day effects [see (Sheth et al., 2008)]. Two additional studies that formally tested for an influence of a time window did not demonstrate such a result. In Doyon et al. 
(2009), the immediate and delayed (5h) post-training napping conditions exhibited comparable subsequent offline changes in explicit motor sequence performance. Furthermore, Schönauer and collaborators (2015) showed similar performance improvements when sleep occurred immediately or up to $24 \mathrm{~h}$ after initial training of an explicit sequence task. These findings are consistent with numerous additional studies - although admittedly not designed to test for an effect of sleep time window - that reported similar performance improvements in both fine and gross motor learning tasks irrespective of the timing of the sleeping episode [i.e., similar post-sleep improvement when sleep occurred immediately vs. $12 \mathrm{~h}$ after learning (Blischke et al., 2008; Genzel et al., 2012b; Kempler and Richmond, 2012; Korman et al., 2003; Lewis et al., 2011; Malangré et al., 2014; Spencer et al., 2007, 2006; Walker et al., 2003)] $]^{1}$. It is important to note, however, that there have been specific examples of a timedependent window for sleep-facilitated consolidation. Consolidation of motor sequence learning by observation, in contrast to active practice, was shown to require an early sleep window [i.e., sleep occurring $12 \mathrm{~h}$ after initial observation did not improve performance at retest (Van Der Werf et al., 2009)]. Similarly, Schönauer and collaborators (2015) showed that sleep had to occur during a certain time window for the consolidation of motor adaptation, and mirror tracing in particular. The authors speculated that the neural networks recruited during mirror tracing may have a lower capacity to maintain the memory trace during wakefulness and therefore require an early sleep window for consolidation to occur (Schönauer et al., 2015).

Altogether, the extant literature suggests that sleep does not have to occur in a specific time window

\footnotetext{
${ }^{1}$ Although all these reported studies employed tasks that fall under the broad umbrella of motor sequences, it is nonetheless important to distinguish between task variants. Participants in these experiments completed explicit motor sequence tasks of the fingers (Blischke et al., 2008; Korman et al., 2003; Spencer et al., 2007, 2006; Walker et al., 2003), an implicit, contextual-dependent finger sequence task (Spencer et al., 2007, 2006), bimanual (Kempler \& Richmond, 2012) or unilateral (Malangre et al., 2014) sequences of arm movements, a sequence of dance movements (Genzel et al., 2012) and a temporal finger tapping sequence task (Lewis et al., 2011).
} 
after initial motor sequence learning to trigger efficient consolidation processes. Whether this is also true for other type of tasks necessitates additional research.

\subsubsection{Does sleep help protect a learned skill from competing material?}

Motor memory consolidation is not only characterized by enhancement (or maintenance) in performance over an interval including no further practice (see Section 2.1.), but also by a reduction in fragility of a motor memory trace after its initial acquisition [see (Robertson, 2012) for review]. The consolidation of a newly learned motor skill (i.e., skill A) can indeed be disrupted if an individual is trained on a competing task (i.e., skill B) immediately after initial learning. This is commonly referred to as retroactive or retrograde interference, as the learning of a task B interferes with the consolidation of the previously learned task A. Importantly, a post-training time-window of approximately 4 to 6 hours for sensitivity to such retrograde interference has been described for both motor sequence learning [e.g., (Korman et al., 2007; Walker et al., 2003), but see (Goedert and Willingham, 2002)] and motor adaptation [e.g., (Brashers-Krug et al., 1996; Krakauer et al., 2005; Shadmehr and Brashers-Krug, 1997), but see (Caithness et al., 2004; Goedert and Willingham, 2002)]. Surprisingly, research investigating whether post-learning sleep influences interference processes is scarce. An earlier study using explicit motor sequence learning showed that a napping episode immediately after initial learning effectively protected the memory trace against the deleterious effect of retroactive interference caused by a competing task (Korman et al., 2007). In a subsequent follow-up examination that again employed an explicit sequence learning paradigm, the protective effect of napping against interference was only shown to be transitory [i.e., within the same testing day, as any group-level differences were abolished after a full night of sleep, see (Albouy et al., 2016)]. These findings suggest that post-learning sleep may play a role in the short-term (i.e., same day) reduction in fragility of an explicit motor sequence memory 
trace, but it is unclear whether such a benefit extends over longer time scales.

The preceding paragraph highlighted instances when a second and competing motor task was used to potentially interfere with an earlier learned motor skill. Interestingly, these interference effects are not strictly limited to within the motor system. In a seminal line of research, Brown \& Robertson (2007) demonstrated that a declarative memory task interfered with procedural memory consolidation (i.e., implicit sequence learning), and vice versa, over wake but not over sleep. The results suggest that sleep can help protect a memory from potential interfering effects even if the two tasks differ in nature.

\subsubsection{Does post-learning sleep facilitate or hinder subsequent performance on a related skill?}

There is limited evidence to suggest that sleep facilitates knowledge transfer from learned to unlearned material. Specifically, sleep has been shown to favor the consolidation of previously learned - as compared to new - sequential pattern in a variety of tasks including implicit sequential tracking (Maquet et al., 2003), implicit serial oculomotor sequence learning (Albouy et al., 2008, 2006) and explicit finger sequence learning (Korman et al., 2003). However, an elegant and recent study has shown that transfer effects could be facilitated by sleep in certain cases [i.e., when learning takes place by imagination and with a particular practice schedule, see (Debarnot et al., 2015)]. The specific combination of (a) variable as compared to constant - motor sequence practice by imagination and (b) subsequent sleep facilitated between-sequence (i.e., learned/unlearned) transfer processes at retest. It is tempting to speculate that the benefits of variable practice on transfer processes might be attributed to the development of generalized motor "schemas" that are known to favor transfer processes (Schmidt, 1975). The link between sleep and the development of motor schemas will also be discussed in Section 2.2.5.3. Finally, although research is relatively scarce, the transfer of grosser motor sequential knowledge (from one dancing choreography to another) seems to depend on time rather than sleep per se (Genzel et al., 
2012b).

Altogether, it appears that sleep facilitates the consolidation of specifically learned motor sequential material and therefore does not simultaneously facilitate knowledge transfer from learned to unlearned material (except under certain circumstances that will be developed further in the next sections). Based on these observations, it has been argued that sleep would favor proactive interference processes (i.e., that the specific knowledge of a consolidated skill would interfere with the learning of a new skill after sleep, but not after wakefulness). A recent study by Borrogan and colleagues (2015) exploring the effect of sleep on proactive interference failed, however, to report such effect. This lack of a sleep-dependent result may not be that surprising given that the consolidation of this task variant (i.e., traditional implicit serial reaction time task) may not be sleep-dependent (Section 2.1.1).

\subsubsection{Influence of pre-sleep performance levels}

There is evidence to suggest that motor sequence performance levels achieved at the end of initial training session - and prior to a sleep / wake interval - influence subsequent sleep-dependent consolidation (Albouy et al., 2008; Wilhelm et al., 2012). Such a result also appears to extend to children (Wilhelm et al., 2012) and older adults (King et al., 2016). It is tempting to speculate that the lack of a sleep effect on the consolidation of tasks more difficult to encode (e.g., probabilistic sequence, gross motor adaptation) could be attributed to an insufficient amount of practice during training as compared to more easily learned tasks (e.g., deterministic sequence, mirror tracing; see Section 2.2.5.5 below for a discussion on task complexity and sleep-facilitated consolidation). This explanation, however, is hypothetical and future studies should manipulate the amount of practice, and thus the performance levels achieved, during initial training in order to assess the influence on sleep-facilitated consolidation in these more complex or difficult tasks. 


\subsubsection{What does sleep consolidate?}

As reviewed above, it appears that the effect of sleep on the consolidation of motor learning is not systematic across all motor tasks and protocols. This raises the question of why the consolidation of some tasks (or task features) requires sleep whereas the consolidation of others does not. This issue has received increased attention in the last decade. In this section, we will review studies investigating the boundary conditions for sleep-dependent motor memory consolidation. Similar to preceding sections, research into this question has predominantly employed motor sequential tasks.

\subsubsection{Consolidation of sequential information}

It has been posited that a boundary condition for sleep-dependent memory consolidation is the presence of sequential information in the motor engram (Blischke et al., 2008). This suggestion is in line with behavioral studies showing sleep-dependent improvement of performance on sequential, as compared to random, material (Robertson et al., 2004b; Spencer et al., 2006). This is also supported in part, by neuroimaging data showing "reactivation" of sequence learning-specific brain regions during post-training sleep [(Peigneux et al., 2003), see Section 3] as well as the established role of the hippocampal system - known to be involved in sleep-dependent consolidation - in the processing of sequential information (Ergorul and Eichenbaum, 2006; Kalm et al., 2013; Kumaran and Maguire, 2006). However, given that the consolidation of traditional implicit sequence learning tasks has not consistently been shown to be sleep-dependent (Borragán et al., 2015; Nemeth et al., 2010; Robertson et al., 2004b; Song et al., 2007; Urbain et al., 2013) and that there are multiple instances of sleep-dependent consolidation in non-sequence tasks such as motor adaptation (Albouy et al., 2013d; Backhaus and Junghanns, 2006; Huber et al., 2004; Javadi et al., 2011; Mantua et al., 2015; Plihal and Born, 1997; Schönauer et al., 2015; Seeck-Hirschner et al., 2010), it is reasonable to conclude that sequential 
information is not a sufficient criterion to trigger sleep-dependent consolidation processes.

\subsubsection{Consolidation and extraction of explicit knowledge}

The idea that motor memory consolidation processes are influenced by the awareness of the material to learn was first introduced by Robertson and collaborators (2004). Using both implicit and explicit versions of a serial reaction time task, the authors showed that explicit sequence learning undergoes sleep-dependent consolidation processes whereas consolidation of the implicit version of the task is time-dependent. These results were predominantly confirmed in a subsequent study from another group (Spencer et al., 2006), although it should be again emphasized that sleep has nonetheless been shown to benefit implicit motor sequence learning under certain experimental conditions (e.g., see Sections 2.1.1.2). Sleep appears to not only consolidate motor sequence learning that has been acquired explicitly, but sleep also favors the extraction of sequence awareness (i.e., explicit knowledge) when the sequence was introduced to the participants in an implicit manner. Fischer and collaborators (2006) demonstrated that when implicit sequence learning was followed by sleep, as compared to wakefulness, there was an increase in explicit sequence knowledge during retest,. This effect was also confirmed in a later study (Wilhelm et al., 2013) and more recently has been shown to be enhanced by Targeted Memory Reactivation (TMR, see Section 4.2.1) during the post-learning sleep episode (Cousins et al., 2014; Diekelmann et al., 2016).

\subsubsection{Consolidation of abstract information}

The acquisition of a new motor skill requires learning different aspects of the task simultaneously. It has been proposed that sequence learning encompasses at least two processes that have been named "spatial" and "motor" (Hikosaka et al., 2002). The spatial representation of learning, also referred to as the perceptual (Nemeth et al., 2009), abstract (Grafton et al., 1998) or effector-independent 
representation (Bapi et al., 2000), represents the goal of the series of movements that need to be executed under allocentric (Cohen et al., 2005) or extrinsic (Witt et al., 2010) coordinates (i.e., in an external frame of reference). By contrast, the motoric representation (Willingham, 1999) constitutes a more intrinsic (Witt et al., 2010), effector-dependent (Grafton et al., 1998) and movement-based skill realized under egocentric coordinates (Cohen et al., 2005). Cohen and collaborators (2005) were the first to introduce the idea that these distinct aspects of an implicit motor sequence task could undergo different consolidation processes that differentially depend on sleep and wakefulness. In their influential study, the authors showed that the consolidation of the motoric representation is wake-dependent, whereas the consolidation of the spatial component depends on sleep. These results have been confirmed and extended in later studies using similar designs and showing that not only nocturnal (PaceSchott and Spencer, 2013) but also diurnal sleep (Albouy et al., 2015, 2013a) enhances the consolidation of a goal-based (spatial), but not a movement-based (motor), representation for both implicit (PaceSchott and Spencer, 2013) and explicit (Albouy et al., 2015, 2013a) sequence learning. Moreover, there is evidence to indicate that the enhanced consolidation of the goal-based representation over sleep intervals may actually hinder the consolidation of the movement-based representation overnight, suggesting that the consolidation processes of these two representations interact (Pace-Schott and Spencer, 2013). In line with these observations, it has also been shown that sleep favors the transfer of the extrinsic (spatial) (Witt et al., 2010), but not the intrinsic (motor) (Hallgató et al., 2012), representation of explicit motor sequence learning.

Interestingly, this link between the abstract/spatial representation of motor sequence learning and sleep-related consolidation processes has been corroborated and extended by several studies using a variety of behavioral paradigms. First, sleep-related consolidation processes have been described to 
promote the development of effector-independent representations of explicit sequence learning [e.g., sequence knowledge that can be transferred between hands, (Korman et al., 2003)]. Second, results from motor imagery and observation studies have shown that imagined or observed sequential movements - that are therefore effector-independent by definition - are also consolidated by sleep (Debarnot et al., 2009; Van Der Werf et al., 2009). Third, consolidation of the perceptual aspect of sequential learning (e.g., monitoring a temporal sequence) is facilitated by sleep (Lewis et al., 2011; Verweij et al., 2016). Fourth, results from Song and colleagues (2014) indicated that sleep preferentially favors the consolidation of ordinal (i.e., the position of the elements within the sequence) rather than transitional (i.e., the series of actual motor movements to perform) properties of motor sequence learning. Ordinal learning consists of binding an event to a spatio-temporal context and is therefore described as a goal-oriented process (Song and Cohen, 2014). Fifth, the manipulation of the practice schedule, thought to favor the development of abstract motor "schema" during initial training (Schmidt, 1975), triggers sleep-related consolidation processes following explicit motor sequence learning (Debarnot et al., 2015). Last, it has been shown that sleep-related consolidation can be boosted when sounds associated with the sequence learning process are presented during post-training sleep [(Antony et al., 2012; Cousins et al., 2016, 2014; Schönauer et al., 2015); see Section 4.2.1] ${ }^{2}$, suggesting that the perceptual component of learning can be reinforced during sleep.

Altogether, results from different research groups and with different experimental manipulations converge toward the notion that sleep consolidates the abstract, effector-independent representation of the motor sequences to perform, an effect that has been observed across both explicit and implicit learning paradigms. Whether the link between consolidation of abstract representations of learning and

${ }^{2}$ Whereas all cited studies employed motor sequence paradigms, Antony et al. (2012) employed an explicit, melodic sequence task. Cousins et al., (2016, 2014) used implicit SRT tasks and Schönauer and colleagues (2015) adopted an explicit motor sequence protocol. 
sleep is unique to motor sequences or whether it is a general process observed across various motor tasks still remains unknown and should be investigated further.

\subsubsection{Consolidation of relevant information}

Fischer \& Born (2009) showed that sleep-dependent consolidation is greater for explicitly learned motor sequences that were associated with monetary reward during training, an effect that was not observed after wakefulness. This result suggests that sleep preferentially consolidates motor sequence memories that have been reinforced, or "tagged" (Frey and Morris, 1997; Moncada et al., 2015) as relevant during initial training. The same group demonstrated that the expectancy of an explicitly acquired motor sequence will be retested following a night of sleep (even without associated reward) also enhanced sleep-related consolidation processes (Wilhelm et al., 2011). However, this latter finding was not replicated in a more recent investigation using an explicit MSL paradigm (Wamsley et al., 2016); thus, additional research is still necessary.

\subsubsection{Consolidation of more complex motor tasks}

The effect of sleep on motor memory consolidation also seems to be influenced by the complexity of the learned motor tasks. Kuriyama and colleagues (2004) demonstrated larger overnight enhancements in performance when the motor sequence that was explicitly learned was longer and performed bimanually, as compared to a shorter and unimanual sequence. A motor imagery study confirmed this finding and showed greater sleep-dependent enhancement in performance for more complex motor sequences involving bimanual coordination (Debarnot et al., 2012). In the same vein but using implicit motor sequence learning, greater enhancements in performance were observed overnight for bimanual, as compared to unimanual, responses and for probabilistic rather than deterministic sequences (Meier and Cock, 2014). Last, although not testing for complexity per se, a sleep-facilitated effect was observed 
with a first person shooter video game employed to represent a more complex sensorimotor skill (Brawn et al., 2008). Altogether, these results suggest that sleep favors the consolidation of more complex motor behaviors, particularly in the context of sequence learning. Similar research with other motor tasks is unfortunately sparse.

\subsubsection{Summary: characteristics of sleep-dependent motor memory consolidation}

Altogether, studies reviewed in this section show that the effect of sleep on motor memory consolidation varies based on specific task features. Whereas results are heterogeneous for motor adaptation and continuous tracking tasks, sleep seems to consolidate features of motor sequence learning, including explicit, abstract and complex information. Interestingly, all these features have been shown or are thought to depend on the hippocampus, a point that will be the focus of Section 3.1.3. Last, sleep appears to not only enhance consolidation of these specifically learned sequential movements, but also to temporarily protect them against the negative effect of interference mediated by competing declarative and motor memories.

- INSERT INSET 2 ABOUT HERE -

\section{Influence of sleep on the neural correlates of motor memory consolidation}

The neural correlates of sleep-related motor memory consolidation processes have been investigated with different techniques, including PET (Positron Emission Tomography), fMRI (functional Magnetic Resonance Imaging), MEG (Magnetoencephalography) as well as non-invasive brain stimulation (NIBS). With neuroimaging techniques (PET, fMRI and $\mathrm{MEG}$ ), these neural correlates can be investigated 
directly, with the measure of cerebral activity during post-learning sleep, but also indirectly with the measure of changes in task-related brain activity after periods of sleep (as compared to wakefulness). NIBS techniques have been used to enhance or disrupt activity in specific cortical areas at different time points during the learning and consolidation process in order to infer the functional role of the specific target. The section that follows will merge the results from these different techniques in order to provide a review of the neural correlates of sleep-related motor memory consolidation. Consistent with the earlier sections, the majority of research to date has employed motor sequential tasks; however, we will briefly detail the small subset of studies that investigated the neural underpinnings of sleep-facilitated consolidation following motor adaptation and continuous tracking.

\subsection{Motor sequence learning}

As results from Section 2 presented above suggest that sleep-dependent effects are observed for explicit as well as specific task variants of implicit motor sequence learning tasks, the following section will collapse across these two paradigms (but will note the type of task employed for the purposes of completeness). It is also worth mentioning that the brain areas discussed in the following section were shown to be specifically linked (or not) to sleep-dependent consolidation processes. Therefore, this section does not discuss the involvement of areas known to play a role in learning and consolidation processes but for which the effect of sleep has not been revealed [e.g., the cerebellum and the parietal cortex; see (Doyon et al., 2009a)].

\subsubsection{Primary motor cortex (M1)}

While the role of M1 in long-term motor memory retention is well characterized [e.g., (Karni et al., 1998)], the specific influence of sleep on this process is less clear. Some studies have shown that both M1 task-related activity (Steele and Penhune, 2010) and learning-related changes in resting-state 
functional connectivity (Gregory et al., 2014) are correlated with next-day improvements in performance in temporal synchrony and explicit motor sequence tasks, respectively. However, the specific effect of sleep in this process was not tested in these studies. Research formally testing for this effect reported discrepant results. Using explicit motor sequence paradigms, increases in M1 activity were observed after both sleep and wakefulness (Fischer et al., 2005); however, other studies reported a sleepdependent increase in activity (Walker et al., 2005) as well as a significant relationship between changes in M1 activity and the amplitude of post-learning sleep spindles (Barakat et al., 2013) - which are known to participate in sleep-dependent memory consolidation [(Fogel and Smith, 2011); see Section 4]. The results of NIBS studies converge on the idea that M1 does not support sleep-dependent consolidation processes. For example, Robertson and colleagues (2005) applied transcranial magnetic stimulation (TMS) over M1 after implicit motor sequence learning and showed that M1 inactivation through TMS disrupted overday, but not overnight, consolidation processes, a result that was subsequently confirmed by another group in an explicit task variant (Hotermans et al., 2008). In line with these observations, performance enhancements on a sequential visual isometric force pinch task triggered by M1 transcranial Direct Current Stimulation (tDCS) during task practice developed over time rather than over periods of sleep (Reis et al., 2015). It is worth acknowledging that all three of these NIBS studies employed the stimulation in the wake state; thus, any conclusion about the impact of such interventions on sleep-dependent consolidation is indirect. Nonetheless, these results are in line with our recent findings showing that M1 supports the motoric representation of learning (Albouy et al., 2015), the consolidation of which does not depend on sleep [see previous section and (Albouy et al., 2015, 2013a; Cohen et al., 2005; Cohen and Robertson, 2007; Hallgató et al., 2012; Witt et al., 2010)]. The available literature thus collectively suggests that M1 does not play a prominent role in sleep-dependent motor 
sequence memory consolidation processes.

\subsubsection{Striatum}

The role of the striatum in motor sequence memory consolidation is well established [see (Doyon et al., 2009a) for a review]. There is also increasing evidence that the striatum is involved in sleep-related consolidation of motor sequence memories. Specifically, striatal activity (Albouy et al., 2008) and functional connectivity (Albouy et al., 2013c) during initial implicit and explicit motor learning, respectively, has been shown to be critical for the subsequent emergence of performance gains observed after sleep as compared to wakefulness. Furthermore, greater striatal activity and striatocortical connectivity were observed in retest sessions with explicit motor sequence task variants following a night of sleep as compared to after similar periods of wakefulness (Debas et al., 2014, 2010; Walker et al., 2005). Three studies extended these findings by showing that these sleep-related increases in striatal activity were linked to post-training NREM sleep spindle activity (Barakat et al., 2013; Fogel et al., 2014) and SWS duration (Cousins et al., 2016) for explicit and implicit MSL tasks, respectively. Moreover, our most recent neuroimaging study suggests that striatal activity participates in motor memory stabilization (i.e., reduction of vulnerability to interference) following explicit MSL and that this process is transiently facilitated by sleep as compared to wake (Albouy et al., 2016). Finally, an increase in functional connectivity between the striatum and reactivated cortical areas was observed during REM sleep after implicit probabilistic motor sequence learning (Peigneux et al., 2003). One should note that this latter result must be interpreted with caution as the consolidation of such a traditional version of implicit motor sequence learning is not thought to depend on sleep (see Section 2.1.1.2.) and that no waking control group was included in this study (but see Section 3.1.5. for further discussion).

On the other hand, a series of studies have demonstrated that striatal activity also increases after the 
simple passage of time, irrespective of sleep or wakefulness. Indeed, striatal activity has been shown to increase monotonically during the day (i.e., 30 minutes and 5 hours post-training), reaching significant and maximal activity 24 hours after implicit oculomotor sequence learning (Albouy et al., 2008). While concluding about the specific effects of sleep versus time per se is impossible with such a time-variant design (see also Inset 2), these findings are consistent with two sleep deprivation studies. Indeed, these studies showed that explicit motor sequence task-related increases in activity in the putamen were evident even when sleep during the first post-training night was prevented (Albouy et al., 2013c; Fischer et al., 2005). It is worth noting though that these studies cannot rule out the possibility that the increase in striatal activity emerged during the sleep afforded during recovery night(s) before retest. However, evidence that the striatum is involved in wake-related processes is also in line with a recent neuroimaging study indicating that the striatum (together with $\mathrm{M} 1$ ) supports the motoric component of explicit motor sequence learning, the consolidation of which does not specifically depend on sleep (Albouy et al., 2015). In summary, in contrast to the numerous studies listed above, this smaller subset of studies suggest that sensorimotor networks including the striatum and M1 are involved in time- rather than sleep-dependent motor sequence memory consolidation processes.

Based on the mixed results regarding the influence of sleep on striatal activity, we argue that the striatum may not directly trigger sleep-dependent consolidation processes, but that it may interact with other brain areas during initial learning and subsequent sleep intervals to further facilitate the consolidation process. This point will be developed further in Section 3.1.3.3 and see Albouy (2013b) for a review. 


\subsubsection{Hippocampus}

3.1.3.1. Hippocampal activity and connectivity during initial motor learning forecasts subsequent sleep-related consolidation

The involvement of the hippocampus in both implicit and explicit motor sequence learning is now well accepted and documented (Albouy et al., 2015, 2012, 2008; Cousins et al., 2016; Fernández-Seara et al., 2009; Fletcher et al., 2005; Gheysen et al., 2010; Lewis et al., 2011; Rieckmann et al., 2010; Rose et al., 2011; Schendan et al., 2003). Moreover, the link between hippocampal activity and sleep-dependent motor memory consolidation processes has received considerable attention, and by our group in particular. Albouy and colleagues (2008) showed that, during initial training of an implicit oculomotor sequence task, learning-related hippocampal activity was correlated with subsequent gains in performance observed overnight, but not over the day (i.e., 30 minutes or 5 hours after the end of training). It was proposed that hippocampal activity during initial training might tag the memory trace to be consolidated during subsequent sleep periods. Interestingly, these data are in agreement with other studies in our group demonstrating that sleep specifically favors the consolidation of an abstract, spatial representation (see Section 2.2.5.3) of an explicitly learned motor sequence (Albouy et al., 2013a), which depends on hippocampo-cortical activity (Albouy et al., 2015). These neuroimaging findings demonstrating a link between hippocampal activity during learning and subsequent sleep-dependent consolidation processes are consistent with numerous behavioral studies that employed variants of motor tasks susceptible to recruit the hippocampal formation. First, implicit sequence learning was improved after a night of sleep only when learning was based on the formation of contextual associations [(Spencer et al., 2006); see Section 2.1.1.2), a process thought to rely on the hippocampus (Chun and Phelps, 1999). Second, sleep preferentially favored the consolidation of ordinal (i.e., the position of the elements within the sequence) properties of a motor sequence (Song and Cohen, 2014), 
the formation of which involves binding between an event and a spatio-temporal context and also thought to rely on the hippocampus (Manns et al., 2007). Third, employing explicit MSL paradigms, sleep has been shown to consolidate relevant information, made pertinent by reward (Fischer and Born, 2009) or expectancy (Wilhelm et al., 2011), a process that has been proposed to involve the recruitment of the prefrontal cortex together with the hippocampus (Miller and Cohen, 2001). Last, sleep contributes to the consolidation of complex (i.e., bimanual vs. unimanual; probabilistic vs. deterministic) motor sequential skills (Debarnot et al., 2012; Meier and Cock, 2014), the development of which necessitates more cerebral control resources in prefrontal regions (Chein and Schneider, 2005), which, together with the hippocampus (Albouy et al., 2013b), code an abstract representation of an explicitly learned sequence. Altogether, these findings indicate that activity in the hippocampus during initial motor learning is a prerequisite to trigger subsequent sleep-related consolidation processes.

Recent studies have suggested that the dynamical activation patterns of the hippocampus as well as its functional connectivity with the striatum were both crucial determinants of subsequent consolidation processes. In particular, we have shown that a competitive functional interaction exists between the hippocampus and the striatum during initial implicit and explicit motor sequence learning, whereby activity in the hippocampus decreases in parallel to an increase in striatal activity and responses in these two structures are anti-correlated (Albouy et al., 2015, 2012, 2008). Critically, this competitive pattern is predictive of subsequent gains in explicit motor sequence performance occurring after sleep, but not after sleep deprivation (Albouy et al., 2013c). In contrast, cooperation between these structures - as illustrated by parallel increases in activity in both structures as a function of initial learning - does not seem to support such processes. Such a cooperative pattern has been observed in elderly subjects (Rieckmann et al., 2010) and young slow learners (Albouy et al., 2008), two participant groups that 
demonstrated no benefit of sleep on motor memory consolidation following implicit (Albouy et al., 2008) and explicit (Fogel et al., 2014) MSL tasks, respectively.

\subsubsection{Task-related changes in hippocampal activity and connectivity after sleep}

There is now increasing evidence that the hippocampus not only conditions subsequent sleep-related consolidation processes, but also that sleep influences subsequent activity in this structure. Indeed, implicit and explicit motor sequence task-related increase of hippocampal activity has been shown to emerge after sleep periods (Albouy et al., 2013c, 2008; Fogel et al., 2014; Lewis et al., 2011; Steele and Penhune, 2010; Walker et al., 2005) - and more precisely in proportion to time spent in SWS (Cousins et al., 2016) but not after periods of wakefulness (Albouy et al., 2008; Fogel et al., 2014; Lewis et al., 2011; Walker et al., 2005) or sleep deprivation (Albouy et al., 2013c). These findings also concur with two studies using intracranial recordings of hippocampal activity in patients with epilepsy that associated the hippocampal formation with sleep-dependent processing of explicit (Moroni et al., 2008) and implicit (De Lucia et al., 2011) $)^{3}$ motor sequence learning. Collectively, results suggest that the hippocampus plays an important role in the retrieval of learned sequences (Ross et al., 2009). Interestingly, it has also been proposed that sleep participates in the reorganization of the functional connectivity of the hippocampus. Albouy et al. (2008) showed that the competitive functional interaction observed between the hippocampus and the striatum during implicit oculomotor sequence learning was transformed into a cooperative interplay after a night of sleep. In line with these results, sleep-related increase in functional connectivity was recently observed between the hippocampus and the caudate nucleus for implicitly learned motor sequences that were reactivated during the post-training night using targeted memory

\footnotetext{
${ }^{3}$ Similar to our discussion of Peigneux et al., 2003 above, results from De Lucia et al., 2011 should be interpreted with caution as the consolidation of such a traditional version of implicit motor sequence learning is not thought to depend on sleep and that no waking control group was included in this study.
} 
reactivation procedures [(Cousins et al., 2016); see also Section 4.2). These findings suggest that not only hippocampal activity, but also its functional connectivity, is reorganized after sleep and perhaps more importantly, reinforces the connection between the hippocampal formation and sleep-related motor sequence memory consolidation processes.

\subsubsection{Hippocampal activity during post-learning sleep}

Reactivation of hippocampal activity during post-learning sleep (i.e., recordings while participants sleep) has long been described to play a crucial role in the consolidation of hippocampal-dependent declarative memory (Peigneux et al., 2004; Rasch et al., 2007). Cerebral activity during sleep following hippocampalmediated motor learning has never been investigated to our knowledge and such evidence is therefore lacking. However, based on the extant literature, it is tempting to speculate that the information developed in hippocampal systems during motor sequence training (i.e., spatial, explicit, ordinal, contextual information) would be reactivated during sleep through hippocampal activity. It has been shown in rats that joint reactivations in both hippocampal and striatal ensembles can occur during sleep (Lansink et al., 2008; Pennartz et al., 2004), and that hippocampal activity can lead ventral striatal replays during consolidation of place-reward associations (Lansink et al., 2009). Based on this evidence, we argue that hippocampal replays during sleep may trigger striatal reactivations and facilitate the consolidation of motor sequence memories in both hippocampo-cortical and striato-cortical networks [see (Albouy et al., 2013b) for alternative hypotheses]. Critically, this interplay between hippocampoand striato-cortical networks overnight may also contribute to the shift from competitive to cooperative interaction between the two networks discussed in the previous section. Nonetheless, it should be stressed that this potential explanation is speculative and should be the focus of future research. 


\subsubsection{Summary: sleep, hippocampus and MSL}

The findings reported in this section highlight the crucial contribution of the hippocampus in sleeprelated motor sequence memory consolidation processes. We have proposed that the hippocampus participates in the learning of motor sequences to perform and that this hippocampal-dependent information will undergo consolidation during sleep, potentially via reactivation processes. Based on the literature reviewed above, it could be speculated that task-dependent hippocampal engagement is a prerequisite for sleep-dependent motor sequence memory consolidation. Thus, a lack of involvement of the hippocampus for a given task may preclude the emergence of sleep-dependent enhancements in performance and thus may contribute to the heterogeneous behavioral findings across various motor tasks (e.g., motor adaptation and continuous tracking) and experimental protocols reported in Section 2. Indeed, although imaging studies are limited, there is no evidence demonstrating a critical involvement of the hippocampus in the learning process for motor adaptation or continuous tracking.

\subsubsection{Prefrontal cortex}

It has been proposed that, together with the hippocampus, the prefrontal cortex codes an abstract representation of the temporal order of the motor sequence rehearsed during learning (Ashe et al., 2006). The decrease in prefrontal activity usually observed as performance becomes more automatic during initial learning has been interpreted as a reduction in the need to consciously regulate and selfmonitor the ongoing movements [see (Albouy et al., 2013b) for a review]. The prefrontal cortex has also been described as a cornerstone in the interaction between the striatal and hippocampal systems during motor sequence learning (Albouy et al., 2012) that is known to condition subsequent sleep-related consolidation (Albouy et al., 2013c).

Specifically regarding consolidation processes, while one group has shown that prefrontal activity during 
explicit motor sequence learning continues to decrease after sleep periods (Fischer et al., 2005), other studies have shown sleep-related increases in prefrontal activity, paralleling hippocampal activity, after sleep periods (Albouy et al., 2013c; Steele and Penhune, 2010; Walker et al., 2005) ${ }^{4}$. We have proposed that, together with the hippocampus, the prefrontal and parietal cortices participate in the building of the spatial map of motor sequences during early learning. This map would be consolidated during subsequent sleep in hippocampo-frontal networks that would in turn be activated again during retest so that the consolidated spatial map of movements can be optimally transformed into fast and accurate movements [see also (Albouy et al., 2013b) for additional hypotheses].

\subsubsection{Cuneus}

In a seminal study, Maquet and collaborators (2000) used PET to record brain activity of participants during both the practice of an implicit serial reaction time task and the subsequent REM sleep episode. Results show that, together with the premotor cortex, the cuneus was re-activated during post-learning REM sleep. Importantly, the same group showed a link between reactivation of the cuneus and the level of performance reached at the end of training on the motor sequential task (Peigneux et al., 2003), suggesting that consolidation-related neuronal processes during sleep are modulated by the learning experience. In the same study, it was also demonstrated that the reactivated cuneus was connected to the striatum during post-training REM sleep. As mentioned earlier, these results might appear surprising as the consolidation of traditional version of the implicit SRT such as the one used in these studies is thought to be sleep-independent (see Section 2.1.1.2). However, a recent study has shown that sleepdependent modulation of cerebral activity can be observed even in the absence of behavioral effects (Urbain et al., 2013). Interestingly, the same study has confirmed that the cuneus is involved in sleep-

\footnotetext{
${ }^{4}$ Albouy et al. (2013c) and Walker et al. (2005) employed explicit motor sequence tasks whereas Steele and Penhune (2010) used a temporal synchronization tapping task.
} 
dependent motor memory consolidation processes, as results demonstrated sleep-dependent modulation of cuneus activity and functional connectivity with the hippocampus after implicit probabilistic motor sequence learning (Urbain et al., 2013). The involvement of the cuneus in sleeprelated motor memory consolidation processes may appear surprising, as it has typically been associated with visual rather than motor processes. However, it is worth noting that the serial reaction time task used in this research includes a highly visuospatial component and that the cuneus is particularly recruited for the processing of spatial attributes (Haxby et al., 1991). Furthermore, and of interest to this review, parts of the occipital cortex including the cuneus have recently been described to code actions at a more abstract level than motor areas (Wurm and Lingnau, 2015). Based on this recent evidence and on the pattern of functional interaction between the cuneus and two crucial nodes of motor memory consolidation processes (i.e., the striatum and hippocampus), it is tempting to speculate that the cuneus together with the hippocampus supports the abstract representation of motor sequences but also interacts with the motoric component in order to optimize the implementation of consolidated movements.

\subsubsection{Premotor cortex, supplementary motor area (SMA) and pre-SMA}

Similar to results observed in the cuneus, Maquet and collaborators (2000) showed that the premotor cortex, which was activated during initial implicit motor sequence learning, was activated again during the subsequent REM sleep episode after which an improvement in motor performance was observed. Follow-up studies have shown that the implicit motor sequence learning experiences taking place during wakefulness modulated not only cerebral activity, but also functional connectivity, of the premotor cortex during sleep. In particular, it was demonstrated that the reactivated premotor cortex was part of a network including the left posterior parietal cortex and bilateral pre-supplementary motor areas 
(Laureys et al., 2001). The involvement of the premotor cortex in sleep-dependent motor sequence memory consolidation has been confirmed in a later study using NIBS. Nitsche and colleagues (2010) showed that tDCS-induced excitation of the premotor cortex during post-learning REM sleep enhanced consolidation processes on an implicit serial reaction time task (however, see discussion on implicit SRT and sleep in Section 3.1.5.). In line with these results, a recent study has shown that implicit MSL-related activity in the premotor cortex was correlated with REM sleep duration during the post-training night (Cousins et al., 2016). Another group also showed decreased activity in the premotor cortex during performance of an explicit MSL task after sleep, interpreted as a reduced need to monitor and control movements after consolidation (Fischer et al., 2005). Finally, the premotor cortex has recently been shown to play a role in the sleep-facilitated process of resistance to interference observed over the course of explicit motor sequence memory consolidation (Albouy et al., 2016). Regarding the role of the SMA in sleep-dependent consolidation process, a recent MEG study showed increased delta and sigma fast oscillations over this area during post-explicit MSL sleep as compared to a baseline night (Tamaki et al., 2013). Importantly, this increase in activity was correlated with between-session changes in performance (see Section 4.1.2).

Altogether, it appears the premotor cortex together with the SMA and pre-SMA play an important role in sleep-related motor memory consolidation processes. Interestingly, Hikosaka and colleagues (2002, 1999) proposed that the premotor cortex and the pre-SMA were crucial nodes ensuring the communication between sensorimotor and associative networks for the implementation of optimal motor behavior. Specifically, the premotor cortex would ensure, during early learning, the transformation between the different coordinate frames (i.e., spatial and motor), a process coordinated by the SMA and the pre-SMA. Based on the experimental evidence described above and the role of the 
premotor cortex during early learning, we argue that the coordinate transformation maps developed in the premotor cortex during early sequence learning are consolidated by sleep to optimize overnight motor sequential behavior.

\subsubsection{Summary: sleep and the neural correlates of MSL}

In conclusion, sleep appears to differently influence brain activity depending on the nature of the information supported by each particular brain area (see Figure 1). In line with our recent work (Albouy et al., 2015), it appears that abstract representation of motor sequence learning, supported by hippocampo-cortical networks, requires sleep for optimal consolidation. In contrast, the motoric representation of motor sequence learning, supported by sensorimotor networks including M1 and striatum, seems to undergo time-dependent consolidation processes. These observations suggest that activity in sensorimotor networks does not necessarily trigger sleep-related motor sequence consolidation processes but does not exclude the possibility that sleep could facilitate consolidation of the motoric representation through functional interaction with other sleep-dependent cerebral networks. We suggest that the between-representation transformation maps developed during initial training in the premotor as well as in the prefrontal cortices undergo sleep-dependent consolidation and facilitate the interaction between the sensorimotor and abstract representations of motor sequence learning over sleep intervals in order to optimize motor behavior. This explanation, however, is speculative and necessitates further investigation.

- INSERT FIGURE 1 HERE - 


\subsection{Motor adaptation}

Although research is relatively sparse, a few studies have investigated the neural correlates of sleeprelated consolidation following motor adaptation. Utilizing $\mathrm{fMRI}$, Debas et al. (2010) reported no differences in cerebral activity between an overnight sleep group compared to wake controls, a result that was in line with the lack of differences observed at the behavioral level. In a subsequent experiment, participants who were sleep-deprived after an initial adaptation session exhibited increased activation in cerebello-cortical networks during a 3-day post-test (Albouy et al., 2013d). The authors argued that the sleep-deprived participants, who exhibited deteriorated performance relative to the end of initial adaptation, were more dependent on substrates typically involved in earlier learning stages. This potentially suggests that post-learning sleep facilitated a reorganization of task-dependent activity. Last, previous research with polysomnographic recordings during post-learning sleep demonstrated that both slow wave activity and sleep spindles were altered in frontal and parietal cortices [(i.e., task-specific regions known to be involved in acquiring a novel visuomotor mapping); (Doyon et al., 2009a)] following the initial learning session (Huber et al., 2004; Landsness et al., 2009; Tamaki et al., 2009, 2008). Moreover, the increase in slow wave activity in the parietal cortex was significantly correlated with overnight performance improvements (Huber et al., 2004; Landsness et al., 2009).

\subsection{Continuous tracking}

To our knowledge, only one study to date has investigated the neural substrates underlying sleep-related consolidation following a continuous tracking task (Maquet et al., 2003). Results revealed that those participants who experienced a night of sleep, as compared to sleep deprivation, following initial learning of a sequential tracking task demonstrated: (a) increased activation in the superior temporal sulcus; (b) increased connectivity between the dentate nucleus of the cerebellum and the superior 
temporal sulcus; and, (c) increased connectivity between the supplementary and frontal eye fields during a tracking retest session 3 days later. The authors suggested that post-learning sleep facilitated activity and connectivity in regions critical for the eye movements necessary to effectively perform the manual tracking task. Moreover, these results build on top of the findings from the MA and MSL literature showing sleep-dependent plasticity in task-dependent neural networks.

\section{Sleep features involved in motor memory consolidation processes}

To describe the sleep features involved in motor memory consolidation processes (see Inset 3 for a description of sleep assessment methods and sleep architecture), we will first review studies examining the relationship between motor memory consolidation and sleep characteristics / stages. We will subsequently review experimental work providing a more causal link between sleep and motor memory processes, i.e., studies in which sleep and its features are actively manipulated via targeted memory reactivation, brain stimulation or pharmacological interventions during the post-training night.

- INSERT INSET 3 ABOUT HERE -

\subsection{Relationship between sleep features and motor memory consolidation}

\subsubsection{Motor sequence learning}

Similar to Section 3 above, the following section will predominantly collapse across implicit and explicit motor sequence paradigms (but will note the type of task employed for the purposes of completeness).

\subsubsection{NREM - stage N2 sleep}

Walker and colleagues (2002) were the first to demonstrate a preferential role of N2 sleep in motor memory consolidation processes using an explicit MSL task. The authors reported a correlation between 
improvements in performance and the percentage of N2 sleep, an effect that was especially prominent in the last quarter of the night. Several subsequent studies, using explicit sequence tasks, also demonstrated a similar relationship between consolidation processes and N2 sleep parameters (Bottary et al., 2016; Korman et al., 2007; Nishida and Walker, 2007; Tucker and Fishbein, 2009). Perhaps most interestingly, the consolidation of the abstract representation of an explicitly acquired motor sequence tended to correlate with time spent in stage N2 sleep during the post-training night (Witt et al., 2010). As reviewed in Sections 2 and 3 above, such an abstract representation of a sequence is thought to be dependent on a hippocampo-cortical network that appears to be tightly linked to sleep-dependent consolidation processes (Albouy et al., 2015).

Using a partial sleep deprivation paradigm (see Inset 3 for details), Genzel et al. (2009) deprived participants of SWS or REM sleep as compared to a night of undisturbed sleep. Neither SWS nor REM sleep deprivation had an effect on consolidation following an explicit motor sequence learning paradigm. The authors therefore concluded that motor sequence learning is predominantly dependent on N2 sleep. Debarnot et al. (2011) reported similar findings, as both short (including at least 10 minutes of N2 sleep) as well as long nap intervals (including both SWS and REM sleep) resulted in similar improvements on an explicit motor sequence learning task. These data suggested - albeit indirectly - that N2 sleep facilitates motor memory consolidation independent of SWS or REM sleep.

\subsubsection{NREM - stage N3 sleep, SWS (S3 and S4)}

Tucker \& Fishbein (2009) were the first to suggest a preferential involvement of SWS in motor sequence memory consolidation processes. They demonstrated that the first 3.5 hours of sleep, which are dominated by SWS, conferred similar performance enhancement on explicit MSL as when additional hours of sleep (total of 7.5h) were afforded. In line with these results, Wilhelm and colleagues (2011) 
described an increase in post-training NREM S4 duration when there was expectancy of a retest, a process that was shown to trigger supplemental overnight gains in performance. Further, an elegant study by Tamaki et al. (2013) showed that oscillations in the delta $(0.5-4 \mathrm{~Hz})$ frequency range (as well as fast-sigma $(14 \mathrm{~Hz})$, see next section) in the SMA contralateral to the trained hand not only significantly increased during post-training SWS, but were significantly correlated with post-sleep performance improvements on an explicit sequence task. The authors suggested that delta oscillations operate to connect or disconnect distant brain regions in order to ensure global plasticity during the consolidation process.

Altogether, the majority of the available literature suggests that SWS plays a critical role in explicit motor sequence memory consolidation processes [but see (Genzel et al., 2009) for negative results]. These results have largely been confirmed but also extended to implicit MSL with recent targeted memory reactivation studies that will be described in detail in Section 4.2.

\subsubsection{NREM - Sleep spindle activity}

Sleep spindles, a hallmark of NREM and known to be critical in declarative memory consolidation processes [for a review, see (Lüthi, 2013)], have also received considerable attention in the procedural memory domain. Correlations between post-learning sleep spindle measures (i.e., density, amplitude, number, sigma power, see Inset 3 for more information) and motor sequence memory consolidation were first detailed by Tucker and colleagues (2009). Overnight performance gains were significantly correlated with the absolute power in both the sleep spindle (sigma, 12-15 Hz) and theta $(4-8 \mathrm{~Hz})$ frequency ranges during the first half of a night of sleep. The relationship between spindle activity and motor memory consolidation also appears to be specific to the learned material. The difference in spindle density in the learning hemisphere (i.e., contralateral to the hand that performed the motor task) 
and the non-learning hemisphere was correlated with between-session improvements on an explicit motor sequence task (Nishida and Walker, 2007). Subsequent research has continued to build on top of these earlier findings by demonstrating not only an influence of sleep spindles on explicit motor sequence consolidation processes (Albouy et al., 2013a; Barakat et al., 2013, 2011; Fogel et al., 2014; Wilhelm et al., 2011), but also an influence of motor learning on spindle activity recorded during the subsequent night (Morin et al., 2008). Specifically, the number and duration of sleep spindles as well as a sigma and beta $(18-20 \mathrm{~Hz})$ power were greater during post-training NREM sleep after explicit motor sequence learning compared to a control task (Morin et al., 2008).

Recently, there has been increased attention to potentially differentiating the precise roles of fast (13$15 \mathrm{~Hz}$ ) and slow (11-13Hz) sleep spindles (but see limitations in Inset 3). Barakat and colleagues (2011) revealed that an increase in fast spindle density was positively correlated with overnight performance. These findings were replicated in a later study of this group (Barakat et al., 2013); however, significant correlations between performance improvements and spindle amplitude were revealed for both spindle types. Interestingly, increases in BOLD signal in the cortico-striatal system (see Section 3 for more details) were correlated positively with the sleep spindle amplitude for both fast and slow spindles (Barakat et al., 2013; Fogel et al., 2014), providing a link between sleep spindles and cerebral activity in MSL-relevant regions. Last, employing simultaneous magnetoencephalography and polysomnography during sleep, Tamaki et al. (2013) provided evidence that the increase of fast-spindle activity (as well as delta activity, see previous section) in the SMA during post-training SWS (but not stage N2 sleep) were correlated with post-sleep performance improvements.

The literature highlighted above suggests a critical role of sleep spindles in motor sequence memory consolidation. However, it should also be noted that the relationship between sequence learning and 
sleep spindle activity can be modulated by a multitude of factors, including the hormones / phase of menstrual cycle in females (Genzel et al., 2012a), the expectancy of motor memory retrieval (Wilhelm et al., 2011) as well as the hand used to perform the task (Cousins et al., 2014; Nishida and Walker, 2007).

\subsubsection{Stage REM sleep}

Maquet and colleagues (2000) were the first to demonstrate a preferential role of REM sleep in (implicit) motor sequence memory consolidation processes. In this elegant study, the authors showed that certain cerebral areas that were active during learning were reactivated during subsequent post-learning REM sleep. Further imaging studies built on top of this seminal research and demonstrated a preferential link between activity in the premotor cortex and REM sleep during consolidation of implicit motor sequence memory [(Cousins et al., 2016; Laureys et al., 2001; Maquet et al., 2000; Nitsche et al., 2010); see Section 3 for more details but also Section 2.1.1.2. for additional discussion on the link between sleep and the consolidation of such implicit motor sequence tasks].

At the behavioral level, offline improvements on an explicit motor sequence task have been related to post-learning REM sleep duration (Fischer et al., 2002); moreover, this effect appears to be specific to the circadian phase in which the REM sleep occurs. Cajochen and colleagues (2004) investigated the circadian modulation of motor sequence learning by the experimental variation of sleep pressure. A constant routine protocol affording the comparison of high and low sleep pressure conditions (i.e., sleep deprivation vs. multiple nights to attain sleep satiation, respectively) was employed. The relationship between REM sleep duration and overnap MSL performance improvements was only found when the nap occurred between 10:00 and -16h:00 (Cajochen et al., 2004). It was concluded that REM sleep only in certain circadian phases contributed to motor memory consolidation (see Section 2.1.1.2. for additional discussion on the link between sleep and the consolidation of implicit motor sequence 
learning). Last, but inconsistent with evidence that the abstract representation of a motor sequence is preferentially linked to NREM sleep [(Albouy et al., 2015, 2013a; Witt et al., 2010); see Section 2], overnight performance improvements on the spatial representation of an implicitly learned motor sequence were positively correlated with the total percentage of REM sleep (Cohen et al., 2005).

In contrast, a series of studies have shown that REM sleep does not play a critical role in motor sequence memory consolidation processes. Korman and colleagues (2007) showed that subjects who failed to reach REM sleep during a post-training nap still showed significant delayed performance gains on an explicit MSL task. In this vein, Tucker and Fishbein (2009) found that the second part of the night, which is dominated by REM sleep, did not support any additional improvements. Further, and as reported above, partial REM sleep deprivation had no effect on explicit motor sequence consolidation (Genzel et al., 2009).

Altogether, the literature is inconclusive with respect to the involvement of REM sleep in motor sequence memory consolidation. Interestingly, the majority of studies showing a relationship between REM sleep and memory consolidation employed an implicit sequence learning task, whereas those exhibiting a relationship between NREM sleep and behavior used predominantly explicit tasks. We are hesitant to put too much emphasis on this observation, as this dissociation has never been explicitly tested. Furthermore, there are numerous instances when no relationships between sleep features and consolidation have been found [e.g., (Albouy et al., 2016; Backhaus et al., 2016; Fischer et al., 2002; Holz et al., 2012; Nettersheim et al., 2015)] and there is evidence based on targeted memory reactivation studies (Section 4.2.1 below) showing a link between implicit learning and SWS. Nonetheless, it is possible that REM sleep may be more critical for implicit motor sequence learning due to the nature of the task. According to Smith and colleagues (Peters et al., 2007; Smith et al., 2004), REM sleep is more 
important for more complex tasks. Given that implicit task versions are often more complex than explicit versions in order to prevent awareness of the repeating sequence [e.g., use of a probabilistic rule (see Section 2.2.5.5)], perhaps REM sleep is more vital for these paradigms. This explanation, however, is certainly speculative.

\subsubsection{Motor adaptation}

In comparison to motor sequence learning, studies investigating the involvement of specific postlearning sleep features in the consolidation of motor adaptation tasks are limited. Huber and colleagues (2004) reported a correlation between local slow wave $(1-4 \mathrm{~Hz})$ activity in the right parietal lobe (a taskrelevant region) during NREM sleep and performance improvements on a visuomotor adaptation task. In addition, there is also evidence indicating that adaptation learning influences characteristics of subsequent sleep. As compared to control conditions, adaptation paradigms have resulted in increased slow wave activity in task-relevant regions (Huber et al., 2004), larger fast sleep spindle amplitude (Tamaki et al., 2009) as well as increased REM density (Fogel et al., 2007).

In summary, there is limited but existing evidence that both REM and NREM sleep play a role in motor adaptation consolidation processes. However, it is important to note that these sleep features have also been shown to have a deleterious effect on consolidation processes. Indeed, employing a gross motor adaptation task (i.e., learning to ride an inverted steering bicycle), Hoedlmoser et al. (2015) revealed that sleep spindle activity and time spent in REM sleep during a post-training nap were negatively correlated with changes in performance. It is worth noting that in this study a midday nap actually hindered performance at retest, a result that was interpreted as sleep serving to protect the more ecologically valid motor memory of riding a normal bicycle (see Section 2). This negative influence of sleep is inconsistent with previous studies that employed fine motor adaptation tasks [e.g., (Albouy et al., 2013d; 
Backhaus and Junghanns, 2006; Huber et al., 2004; Javadi et al., 2011; Mantua et al., 2015; Schönauer et al., 2015; Seeck-Hirschner et al., 2010)].

\subsubsection{Continuous tracking}

In one of the first sleep and motor learning studies, Smith and McNeill (1994) used a variation of four sleep manipulations in comparison to a control night to investigate the impact of different sleep stages on the consolidation of a pursuit rotor task. Results collectively suggested that stage N2 - but not REM sleep was important for consolidation of pursuit rotor learning. A subsequent study expanded on this finding and revealed that N2 sleep spindle density during the night immediately after initial learning was correlated with performance improvements observed one week later (Fogel and Smith, 2006). In line with these results, it has also been shown that learning a continuous tracking task resulted in increased stage N2 sleep, sleep spindle density and duration as well as slow sleep spindle power (Fogel et al., 2007; Fogel and Smith, 2006). Interestingly, the influence of learning on subsequent sleep architecture appears to be dependent on skill levels obtained during the initial training period (Peters et al., 2008). Participants who obtained high levels of skill demonstrated a significant increase in subsequent N2 spindle density; and, spindle density was significantly correlated with performance one week later. In contrast, there was a significant correlation between changes in REM density and performance on the pursuit rotor task during retest one week later in low-skill participants, although the actual increase in REM density failed to reach significance in this group. This finding may also be related to the beneficial effects of REM sleep for the consolidation of more complex tasks discussed in Section 4.1.1.4 above (Peters et al., 2007; Smith et al., 2004).

\subsubsection{Additional tasks}

As described in Section 2.1.4, a subset of studies has examined the effects of sleep features on less 
commonly employed motor tasks. In a three-ball cascade juggling task, performance was shown to improve after a post-training nap and SWS duration was greater after learning compared to a baseline nap (Morita et al., 2012). In addition to these macroscopic changes in sleep architecture, slow oscillation, delta, sigma and slow sigma $(12-14 \mathrm{~Hz})$ power significantly increased during post-training NREM sleep. These EEG power changes were especially dominant during slow wave, but not stage N2, sleep (Morita et al., 2012). Conversely, REM sleep duration was found to increase over a 13-week training on a new and complex trampoline pattern, but not after dancing or soccer (Buchegger et al., 1991). Last, sleep spindle number and sigma power were shown to significantly predict performance on the ball-and-cup task following a nap, but only for those individuals who habitually take naps (Milner et al., 2006). These results indicate a link between sleep features and motor memory consolidation, but there are too few studies to draw any major conclusions.

\subsubsection{Summary: correlation between sleep features and motor memory consolidation}

Taken together and with respect to motor sequence learning in particular, NREM sleep, including N2, SWS and sleep spindles, have been shown to be involved in memory consolidation processes. The picture, however, is less clear for REM sleep. It is important to acknowledge that several studies did report statistically non-significant correlations between specific sleep parameters and motor memory consolidation [e.g., (Albouy et al., 2016; Backhaus et al., 2016; Fischer et al., 2002; Holz et al., 2012; Nettersheim et al., 2015)]. Moreover, there are likely many additional publications that did not report results from correlational analyses if nothing was revealed to be significant; thus, results from this type of approach should be interpreted with caution.

The correlational results highlighted above present somewhat of a blurry picture, as various sleep stages and features have been shown to be related to motor memory consolidation processes. Rather than 
viewing these heterogeneous findings as simply conflicting, it is possible that they represent a complex, multi-stage, sequential memory consolidation process that proceeds over the various sleep stages [e.g., (Sara, 2017; Sonni and Spencer, 2015; Stickgold et al., 2000)]. Specifically, optimal motor memory consolidation may depend on sequential processing that occurs over NREM-REM (or even stage N2 and SWS within NREM) cycles, rather than on one distinct sleep stage or feature. Such a sequential hypothesis has received more attention in the declarative memory domain [e.g., (Sara, 2017; Sonni and Spencer, 2015)] and additional research is certainly necessary in the context of motor memory consolidation.

\subsection{Active manipulation of post-learning sleep}

Although many studies have found robust correlations between behavior and certain sleep features (especially for motor sequence learning, see Section 4.1), the causal relationship between sleep and motor memory consolidation still remains an open question. To address this knowledge gap, researchers have aimed to actively manipulate and modulate sleep features (e.g., sleep architecture, slow oscillations, sleep spindle activity) in order to investigate the causal impact of such modulations on consolidation, as reflected by post-sleep performance. Currently, there are three primary approaches reported in the literature to investigate memory consolidation mechanisms by actively modulating postlearning sleep: (a) olfactory or auditory targeted memory reactivation (TMR), (b) transcranial electrical stimulation (TES), and (c) administration of pharmacological drugs. We will address the effect of each of these manipulations in the subsequent sections. Consistent with the majority of sleep and motor memory literature, the majority of these studies have employed motor sequence learning paradigms.

\subsubsection{Targeted memory reactivation}

A classic way to evoke reactivation of learned material is to link it to specific stimuli during acquisition 
(i.e., conditioning experimental designs). Afterwards, the presentation of the stimuli alone presumably triggers the processing of the associated memory (i.e., triggering a "replay" of the respective neuronal activation patterns). This process, referred to as TMR, can be triggered during post-learning wakefulness as well as sleep [for review, see (Oudiette and Paller, 2013)]. Olfactory or auditory associated stimuli are most commonly used because they can be presented without disrupting ongoing sleep (Carskadon and Herz, 2004). Moreover, odor in particular seems to be a promising stimulus to modulate hippocampusdependent memories, as primary olfactory processing areas directly project to higher-order regions like the hippocampus while circumventing the thalamus (Carlson, 2004). TMR during sleep has previously and extensively been shown to enhance declarative memory (Diekelmann et al., 2011; Guerrien et al., 1989; Rasch et al., 2007; Rihm et al., 2014; Rudoy et al., 2009; Schreiner and Rasch, 2015; Sterpenich et al., 2014; van Dongen et al., 2012). It was only in 2007 that TMR approaches were utilized to investigate motor memory consolidation mechanisms, and with motor sequence learning paradigms specifically. Rasch et al. (2007) presented specific odors during explicit motor sequence learning and re-exposed the subjects to the olfactory cue during either SWS or REM sleep. Neither condition elicited a benefit in motor performance during retest (but see the limitations imposed by such multi-task design in Inset 4). Given the robust relationship between N2 sleep and motor sequence memory consolidation (see Section 4.1.1.3), Laventure et al. (2016) recently investigated the contributing role of N2 sleep - with a specific interest in sleep spindles - on explicit motor sequence learning with a similar olfactory TMR protocol. Results showed that performance at retest was better in the N2 sleep cued group as compared to the REM sleep cued group and as compared to controls (not conditioned). Moreover, N2 cueing led to an increase of parietal sleep spindle frequency, amplitude as well as duration. Taken together, these results provide evidence that olfactory TMR during N2 (Laventure et al., 2016), but not SWS or REM sleep (Rasch 
et al., 2007), effectively influences the microstructure of sleep - and sleep spindles in particular - and thereby enhances subsequent sequential motor performance.

In the same vein but employing an acoustic TMR paradigm, Antony and colleagues (2012) sought to boost the ability to produce a sequence of finger movements associated to a melody that was subsequently replayed (i.e., "cued") during post-training SWS. The results indeed showed that performance was better at retest for motor sequences that were cued, compared to those that were non-cued, during subsequent sleep. Using a similar design, Cousins et al. (2014) showed that not only motor performance, but also the explicit knowledge of the otherwise implicitly learned sequence, at retest was better for motor sequences associated to the melody that was cued during the post-training SWS. Moreover, the number of recalled items was correlated with slow oscillation power. Although the presentation of the tones did not seem to actively increase power during SWS, together with lateralized fast spindle density at central electrodes, SWS power might be predictive for the effectiveness of acoustic cueing. Schönauer et al. (2014) built on top of these findings and found that, while TMR during sleep reduced the duration of SWS (but not total sleep time), it improved later motor performance. Additionally, 3 hours of sleep with reactivation was as beneficial as a whole night's sleep without auditory cues. The authors concluded that the availability of sufficient reactivation during sleep (i.e., via cueing or longer periods of sleep) is important for motor memory consolidation. Finally, a very recent study by Cousins et al. (2016) showed that acoustic TMR during post-training SWS not only enhanced implicit motor sequence performance, but also modulated brain activity and connectivity in consolidation-relevant networks during retest (see Section 3 for more details).

In summary, TMR studies have not only suggested a preferential role of NREM sleep in motor sequence memory consolidation processes, but also offer the unique evidence for a causal relationship between a 
certain sleep feature or stage and motor memory consolidation. Furthermore, earlier research has demonstrated that sensory processing and cortical responses to external stimuli during NREM sleep are strongly influenced by the phase of slow oscillation at which stimuli were delivered. Specifically, the brain is more responsive during the positive slope of the slow oscillation, whereas lower sensory processing was revealed during a spindle and the descending phase of the slow oscillation (Bergmann et al., 2012; Massimini et al., 2003; Schabus et al., 2012). Therefore, future TMR research should focus on this specific time window (positive ongoing phase of slow oscillation) for the stimulation to offer the greatest boost to memory consolidation processes (Oudiette et al., 2013). Note that our literature search did not highlight any TMR studies using motor adaptation or continuous tracking and that future research should focus on these tasks.

\subsubsection{Transcranial electrical stimulation}

An alternative approach to investigate the causal relationship between sleep and motor memory consolidation and to "boost" motor skill learning is the utilization of NIBS techniques. Transcranial electrical stimulation (TES) is one of these techniques (Nitsche and Paulus, 2011) and allows the modulation of brain activity by inducing a low current into the brain via two electrodes (anode and cathode) placed on the scalp. Three main TES techniques - based on the nature of the current administered - are commonly used (Herrmann et al., 2013): (a) transcranial direct current stimulation (tDCS, constant current), (b) transcranial alternating current stimulation (tACS, alternating currents at a certain frequency), and (c) oscillatory tDCS (otDCS, combination of alternating and direct currents). Critically for the purposes of this review, these approaches can be applied during sleep in order to influence cortical excitability in distinct sleep stages.

Marshall and colleagues (2004) were the first to apply frontal anodal tDCS during sleep to boost 
endogenous slow oscillation activity and thereby attempt to enhance sleep-associated memory consolidation. Subjects were trained on both a declarative (word pair association) and a motor task (mirror tracing) and stimulation commenced when subjects reached SWS. tDCS positively affected declarative performance compared to a sham stimulation as well as a wake control group. Interestingly, mirror tracing performance was not affected after tDCS. In subsequent follow-up studies with altered stimulation paradigms (tACS and otDCS), stimulation of the frontal cortex during sleep offered no offline benefits following mirror tracing or explicit motor sequence tasks (Antonenko et al., 2013; Marshall et al., 2011, 2006). In summary, whereas the enhancement of slow wave activity by frontal TES appears to enhance declarative memory consolidation, the influence on motor memory is limited.

In contrast, there is evidence that TES during sleep can offer some benefits if applied in a more selective manner. For example, Nitsche et al. (2010) applied anodal (i.e., excitatory) tDCS over the premotor cortex during the second REM sleep phase after subjects practiced an implicit serial reaction time task. Recall of the learned sequences was significantly improved after anodal - as compared to sham - tDCS immediately after the stimulation but not after a whole night of sleep. Besides highlighting the importance of stimulating task-relevant regions, these results favor the relevance of REM sleep, as anodal tDCS resulted in increased REM density, which in turn showed a trend for a positive correlation with memory consolidation (but see Section 2.1.1.2. for some considerations on sleep-dependent consolidation of implicit motor sequence learning). Second, Lustenberger et al. (2016) effectively boosted motor memory consolidation by employing fronto-central TES during sleep with a novel feedback-controlled, and thus individually-adjusted, stimulation protocol. Specifically, an EEG-feedbackcontrolled approach that employed $12 \mathrm{~Hz}$-tACS and selectively targeted sleep spindles in real time was employed. This stimulation protocol - as compared to a sham condition - boosted subsequent fast sleep 
spindle activity (without modulating other sleep rhythms or sleep architecture) and enhanced sleepdependent consolidation of an explicit motor sequence task. This elegant study not only provides evidence for a causal role of fast sleep spindles in motor memory consolidation, but also suggests that the modulation of brain oscillations during NREM sleep with TES is a promising approach to boost sleepassociated motor memory consolidation.

Note that TES during sleep over the frontal cortex does not appear to enhance consolidation processes for motor adaptation tasks such as mirror tracing (Marshall et al., 2011, 2006, 2004), a result may be attributed to the fact that adaptation is dependent on different neural substrates and thus the stimulation may not have been suited for the particular task. Our literature search did not reveal any TES studies using tracking tasks and future research should address this knowledge gap.

\subsubsection{Pharmacological drug administration}

In order to investigate REM-related reactivation and consolidation mechanisms of explicit motor sequence learning and motor adaptation (i.e., mirror tracing), Rasch et al. (2009) administered two antidepressant drugs known for suppressing REM sleep (i.e., fluvoxamine and reboxetine) in healthy subjects. As expected, both substances, in comparison to a placebo condition, reduced the amount of time spent in REM sleep, increased the amount of time spent awake and in stage N1 sleep, but exerted no influence on SWS. Moreover, reboxetine increased the amount of time spent in stage N2 sleep, as well as sleep spindle number and the density of fast spindles. While mirror tracing performance remained unchanged after drug administration, performance on the MSL task was enhanced. Interestingly, the difference in MSL improvements in accuracy between active treatment and placebo conditions was positively correlated with the change in fast sleep spindle number and density. These results not only support the numerous reports of the positive relationships between spindle activity and 
overnight gains in sequence learning, but also suggest that REM sleep does not seem to facilitate consolidation of motor sequence learning (see also Section 4.1). Nevertheless, the results of studies using pharmacological methods to manipulate sleep-related memory consolidation have to be interpreted with caution as (a) pharmacological REM sleep suppression might not be equal to natural one, (b) the location of action of the administered substances cannot be determined exactly and (c) pharmacological drugs are active throughout the whole sleep period and thus not necessarily specific to a sleep stage or feature.

\subsubsection{Summary: active manipulation of sleep and motor memory consolidation}

Taken together, results from research approaches which directly manipulate sleep stages and features converge on the notion that NREM sleep, including stage N2 sleep and sleep spindles, play a critical role in the motor sequence memory consolidation processes. Moreover, these approaches have been shown to be powerful tools that can enhance motor (sequence) memory consolidation processes at both the behavioral and neural levels. The development of tools to optimize motor behavior certainly offers important applicative value, as similar investigations may be employed to offset age- or pathologyrelated declines in motor performance. But, critically, the results highlighted in this section are remarkably consistent with the literature reviewed in Section 4.1, in which NREM sleep was shown to be correlated with motor sequence memory consolidation processes at the behavioral level.

\subsection{Summary: sleep features involved in motor memory consolidation}

The studies reviewed above converge toward the notion that NREM sleep - and sleep spindles and slowwaves in particular - is critically involved in memory consolidation processes, and motor sequence memory consolidation in particular. In this section, we will attempt to merge the findings reviewed in 
Section 4 on sleep features with the behavioral and neural processes supporting motor memory consolidation processes presented earlier and provide potential cellular mechanisms supporting these processes when applicable. Based on the evidence that sleep spindles are (1) time-locked with hippocampal activity in humans (Andrade et al., 2011; Schabus et al., 2007) as well as hippocampal replay (Fogel and Smith, 2011; Siapas and Wilson, 1998) and hippocampal local field potential features called "sharp wave ripples" (Kudrimoti et al., 1999) in animals; and (2) associated with increased activity within (Clemens et al., 2011; Schabus et al., 2007) as well as functional connectivity between (Andrade et al., 2011) the hippocampus and the prefrontal cortex, it is tempting to speculate that spindle activity supports the consolidation of hippocampal-cortical-mediated motor memory traces. Specifically, the temporal synchronization of hippocampal ripples and cortical spindles is believed to facilitate the transfer of encoded sequential information from the hippocampus to the neocortex (Born et al., 2006). In line with these observations, it has been demonstrated at the cellular level that sleep spindles provoke a massive $\mathrm{Ca}^{2+}$ influx into cortical pyramidal neurons and might therefore "open the door for subsequent long-term changes in cortical networks" (Sejnowski and Destexhe, 2000, p. 220). Similarly, it was shown that the exact pattern of spindle-associated spike discharges can reliably induce long-term potentiation in neocortical neurons in vitro (Rosanova and Ulrich, 2005).

The link between sleep spindles and hippocampal-cortical mediated motor memory traces is consistent with the observation that spindles are involved in the consolidation of abstract, hippocampal-dependent representation of motor sequences (Albouy et al., 2015, 2013a). We propose that the spatial maps of motor actions - and motor sequences specifically - encoded in hippocampo-cortical networks during learning are strengthened during spindle events. It has been previously suggested that hippocampal replay during the night could indirectly trigger the reinforcement of striatal-mediated motor memory 
traces through cooperative interaction between these two memory systems over the night (Albouy et al., 2013b). This notion is also consistent with studies linking sleep spindles to striatal activity (Barakat et al., 2013; Caporro et al., 2012; Fogel et al., 2014; Tyvaert et al., 2008). We thus propose that sleep spindles may be involved in localized plasticity processes (Tamaki et al., 2013) beneficial for the consolidation of abstract hippocampo-cortical representations of learning, which in turn can trigger consolidation of motoric striato-cortical representations. In contrast and based on the suggestion that slow oscillations support longer range global plasticity processes (Tamaki et al., 2013), we argue that slow waves travelling from the frontal cortex to more posterior areas (Massimini et al., 2004; Murphy et al., 2009; Riedner et al., 2011), including the hippocampus (Nir et al., 2011), facilitate the interaction between the sensorimotor and abstract representations of learning (see Figure 1). Altogether, these processes of local and longer-range plasticity would ensure consolidation within and between the specific components of motor learning and forecast optimized motor behavior overnight. This explanation, however, remains hypothetical and certainly deserves more attention in future research.

The picture is far less clear for the physiological processes during REM sleep, which might be important for motor memory consolidation. Based on animal studies by Datta and Patterson (2003) linking REM sleep and synaptic plasticity in rats, Smith and colleagues (2004) proposed that REM sleep duration (i.e., minutes spent in REM sleep) and density (i.e., number of rapid eye movements per REM period) are linked to consolidation processes occurring within the cortico-cerebellar motor system, as REM sleep deprivation leads to cellular changes within the cerebellum (i.e., reductions of brain-derived neurotrophic factor and intra-cellular calcium, which are known to be relevant for synaptic plasticity). Given that the consolidation following motor sequence learning is heavily dependent on the corticohippocampal and cortico-striatal - and less so on the cortico-cerebellar - system [e.g., (Albouy et al., 
2013b; Doyon et al., 2009a)], this may also help explain why REM sleep does not appear to be critical for this specific process.

\section{Conclusions and future directions}

Although some important methodological issues - that need to be considered in future research (see Inset 4) - could, at least partially, explain why the sleep and motor memory picture has been a bit blurry over the last years; we believe that the diversity of results reported in the present paper reflects the multifaceted nature of the sleep-related motor memory consolidation process. The ensemble of studies reviewed here indeed indicates that the positive influence of post-motor learning sleep on memory consolidation is not universally observed. Rather, the effects appear to depend on specific factors, including the characteristics of the motor task, the nature of the learned information, the cerebral areas recruited during learning, as well as the features of post-learning sleep. This view is in line with the "consolidation complexity hypothesis" recently contested (at least with respect to explicit motor sequence learning paradigms) by Rickard and Pan (2017) in response to Adi-Japha and Karni (2016). It is important to note that it is difficult to draw too many comparisons between the results in Rickard and Pan (2017) and our review due to differences in approaches employed (i.e., quantitative meta-analysis vs. qualitative review) as well as the scopes of the two papers (i.e., emphasis on explicit sequence learning only vs. multiple motor tasks and task variants, strictly behavioral results vs. relationships among behavior, neural correlates and specific sleep features). Nonetheless, our exhaustive and qualitative literature review identified that sleep tends to offer benefits following explicit sequence learning, specific variants of implicit sequence learning tasks and mirror tracing visuomotor adaptation. Sleep appears to enhance motor sequence consolidation when the task is more dependent on cortico- 
hippocampal networks; for example when explicit, spatial, abstract, or more complex information is learned. Conversely, the role of sleep in the consolidation of non-hippocampal-mediated tasks remains less clear. Last, NREM sleep, including sleep spindles and slow waves more particularly, appears to be critically involved in sleep-dependent motor sequence memory consolidation processes. Importantly, these specific sleep features can be manipulated during post-learning sleep to optimize motor sequence memory consolidation processes.

Although research over the last 20 years has produced incredible insight into the role of sleep in motor memory consolidation, several critical knowledge gaps remain to be addressed. For example, and as reviewed above, the vast majority of literature to date has examined the influence of sleep on the consolidation following motor sequence learning tasks. Comparatively less is known about sleep's role following other motor learning paradigms, including motor adaptation, continuous tracking or more gross complex and ecological motor tasks, including elite athletic performance [e.g., (Yarrow et al., 2009)]. No study, to our knowledge, has decomposed these tasks in an effort to determine if sleep selectively benefits specific task features. Based on the multifaceted nature of the sleep-related motor memory consolidation process highlighted in this review, we argue that such an effort will help explain discrepant results in the literature. Second, investigations into sleep-dependent consolidation processes across the lifespan (i.e., in children, adolescents and older adults) are of interest due to the robust agerelated changes in both motor memory consolidation [e.g., (Adi-Japha et al., 2014; Ashtamker and Karni, 2013; Brown et al., 2009; King et al., 2013; Nemeth and Janacsek, 2010; Roig et al., 2014)] as well as sleep duration and architecture [e.g., (Landolt and Borbely, 2001; Marcus et al., 1992; McClain et al., 2016; Montgomery-Downs et al., 2006; Ohayon et al., 2004)]. Although considerable progress has been made in this research area [e.g., (Bottary et al., 2016; Desrochers et al., 2016; Fogel et al., 2014; King et 
al., 2016; Sonni and Spencer, 2015; Wilhelm et al., 2013, 2012)], additional research is still necessary to further elucidate lifespan changes in the role of sleep on motor memory consolidation. Third, there has been increased interest recently in memory consolidation processes following the reactivation or retrieval of a previously acquired motor memory trace, a process often referred to as reconsolidation (Censor et al., 2016, 2014; Dayan et al., 2016; de Beukelaar et al., 2016, 2014; Hardwicke et al., 2016; Sandrini et al., 2015; Wymbs et al., 2016). The role of post-reactivation sleep on reconsolidation is still poorly understood and necessitates further investigation, as such reactivations are common when learning a motor skill over multiple days / training sessions. Fourth, future research should attempt to clarify the precise roles of specific sleep features in motor memory consolidation. Based on the literature reviewed above, NREM sleep - with sleep spindles and slow waves in particular - appears to play a critical role in memory consolidation processes, particularly following motor sequence learning but also motor adaptation (Huber et al., 2004) and less commonly employed tasks such as juggling (Morita et al., 2016, 2012). However, whether these sleep features are supporting the consolidation of specific task components, how these features potentially interact to influence consolidation processes, and the relationship between these features and relevant neural networks (i.e., cortico-striatal and corticohippocampal) are all open questions. Finally, we believe that the identification of the specific neurophysiological pre-requisites for sleep-related motor memory consolidation processes will open new and exciting avenues in the field of memory optimization. Modulating these neurophysiological processes [e.g., with brain stimulation, see (Lustenberger et al., 2016)] is an innovative way to optimize the motor memory consolidation process and has high applicative value for populations showing deficits in consolidation processes (e.g., elderly), but also in the context of neurorehabilitation (e.g., after stroke). 
- INSERT INSET 4 HERE - 


\section{Insets}

\section{Inset 1: Motor learning tasks}

The effect of sleep on motor learning has been investigated using a multitude of motor tasks involving different requirements and constraints on the motor system, different effectors as well as different movement amplitudes and levels of complexity. In this inset, we review and classify the three dominant motor tasks (Figure I1) used in research investigating the influence of sleep on motor memory consolidation. Previous research has employed additional tasks that fall outside of these primary paradigms, but these are discussed in the corresponding sections on a case-by-case basis.

Motor sequence learning (MSL) represents the incremental acquisition of sequential movements into a well-articulated behavior (Karni et al., 1995; Nissen and Bullemer, 1987). The most frequently used fine MSL task involves unilateral sequential finger movements according to a short ( 5 to 8 elements in general) and explicitly known sequence provided to the participant prior to practice. The finger movements may be in opposition to the thumb [e.g., (Fischer et al., 2005; Karni et al., 1998)] or tapping on a keyboard [see Figure I1A where subject tap buttons of a response box in a fixed sequential order; e.g., (Albouy et al., 2013a; Walker et al., 2003)]. Note that, even if less frequent, bimanual [e.g., (Kuriyama et al., 2004)], imagination [e.g., (Debarnot et al., 2012)] and observation [e.g., (Van Der Werf et al., 2009)] versions of the tasks can be found in the sleep and memory literature. The learning of sequential finger movements has also been extensively investigated using the Serial Reaction Time Task [e.g., (Robertson et al., 2004b; Song et al., 2007)] that requires the participants to type a unimanual or bimanual sequence of finger movements according to a pattern displayed on the screen (i.e., visually cued as opposed to self-initiated). The pattern to reproduce can be of different nature (e.g., sequential or random), follow different rules [deterministic, see (Robertson et al., 2004b) or probabilistic, see (Song et al., 2007)] and be accessible or not to awareness [explicit or implicit, see (Robertson et al., 2004b)]. Similarly, the learning of sequential ocular movements has been investigated with the Serial Ocular Reaction Time Task [e.g., (Albouy et al., 2008, 2006)]. Although the majority of MSL studies involves finger movements, the effect of sleep on more gross sequence learning has been investigated using simple unilateral or bilateral arm movements in an unconstrained manner [e.g., (Gudberg et al., 2015)] or with constraints applied to the plane of movement [e.g., (Kempler and Richmond, 2012; Malangré et al., 2014)]. Generally, the instructions provided to the participants for all task variants are to move as fast and as accurately as possible.

Motor performance for sequence tasks is most often assessed in terms of speed (e.g., time to perform the task) and accuracy (e.g., number of correct sequences) but can also be measured with combined speed-accuracy scores [e.g., (Dan et al., 2015; King et al., 2016; Rumpf et al., 2017)]. Changes in performance between the end of the initial training session and the beginning of the subsequent retest session (taking place after sleep or wakefulness, for example) are often taken as indicators of consolidation processes. It is to note that the magnitude of these offline (i.e., inter-session) changes in performance must be interpreted carefully as it can be confounded by several factors, including an increase in fatigue as a result of training or the specific computation employed to assess offline change [e.g., number of blocks/trials included; see (Adi-Japha and Karni, 2016; Pan and Rickard, 2015; Rickard and Pan, 2017)].

Motor adaptation (MA) represents the capacity to modify motor behavior in response to changes in the environment or organism [e.g., (Cunningham and Welch, 1994; Shadmehr and Mussa-Ivaldi, 1994)]. The majority of MA paradigms consist of reaching tasks involving unilateral movements of the upper limb 
(i.e., the hand, wrist and/or arm) with various end effectors, including, for example, a computer mouse (Albouy et al., 2013d), joystick (Debas et al., 2010) or robotic manipulandum (Donchin et al., 2002). These changes are applied in laboratory settings in a dynamic or kinematic (shown in Figure I1B) manner in order to perturb the actual (e.g., application of an external force) or perceived movements (e.g., application of a visuomotor rotation), respectively (Robertson et al., 2004a). For example, mirror tracing, a commonly employed version of kinematic motor adaptation, requires participants to execute movements (e.g., tracing a star-shaped pattern with a pen) with only inverted visual feedback of workspace and task performance available through a mirror. In all adaptation task versions, the participants need to compensate for these actual or perceived changes in order to complete the initially intended reaching movement. Participants are instructed to be as accurate as possible while completing the movement within specified temporal or speed constraints. Although less common, the influence of sleep on grosser kinematic MA paradigms has been examined by asking participants to ride a bicycle with an inverted steering wheel (Hoedlmoser et al., 2015).

Performance on MA tasks is generally assessed in terms of speed (e.g., time to reach the target/complete the task) or accuracy (e.g., directional error at peak velocity, number of deviations from desired trajectory, root mean squared error (RMSE)) but can also be measured with combined speedaccuracy scores [e.g., (Debas et al., 2010)]. MA memory consolidation processes have typically been assessed by savings in performance, represented by the change in performance between the first trials of delayed testing relative to initial training [e.g., (Albouy et al., 2013d; Donchin et al., 2002)]. Similar to MSL, the influence of sleep on offline changes in performance between the end of training and start of retest has also been investigated in MA [e.g., (Albouy et al., 2013d; Huber et al., 2004)].

Continuous tracking (CT) tasks include motor learning tasks in which participants continuously track a moving visual stimulus with an end effector [e.g., pursuit rotor task, see (Fogel and Smith, 2006)]. The moving stimulus typically follows a predictable, but sometimes complex, pattern [(Maquet et al., 2003); Figure ${ }_{11 C} \mathrm{C}$ and participants are instructed to track the stimulus as accurately as possible.

Performance is generally assessed in terms of accuracy (e.g., time on target, RMSE) and consolidation processes are most frequently measured via changes in performance between the training and retest sessions [e.g., (Rieth et al., 2010; Smith and MacNeill, 1994)].

- INSERT FIGURE I1 HERE - 


\section{Inset 2: Experimental designs}

A variety of experimental designs have been employed to examine the effect of sleep on motor memory consolidation [Figure 12; also see (Pan and Rickard, 2015)]. Critically, in each of these designs, participants complete an initial learning session of a motor skill and at least one retest following a retention interval that includes diurnal or nocturnal sleep (as compared to waking control groups). First, in an evening/morning design, also called PM/AM design [e.g., (Walker et al., 2002); Figure 12A], participants learn a motor task either in the morning or the evening and then are retested following an interval of 12 hours. Thus, learning is followed by $12 \mathrm{~h}$ of wakefulness or $12 \mathrm{~h}$ including a night of sleep. The advantage of this design is that the sleep vs. wake comparison includes a full night of sleep without disrupting participants' regular sleep schedules. However, it has the obvious disadvantage that the two experimental groups complete both the initial learning as well as the subsequent retests at different times of the day; thus, circadian variations may present a confound. Second, a Nap/No Nap experimental design [e.g., (Korman et al., 2007); Figure 12B] requires participants to complete both initial training and at least the first retest in the same day; the retention interval then includes wakefulness or both diurnal sleep and wakefulness. Critically, in this paradigm, the two experimental groups do not differ with respect to the times of day of the training and retest sessions and these sessions are separated by less than $4 \mathrm{~h}$ in the early afternoon, which thus prevents the potential between- and within-group circadian influences, respectively. The drawback, however, is that the intervening sleep episode consists of only diurnal sleep; thus, sleep duration is obviously shorter, participants may have difficulties falling asleep and later sleep stages such as slow wave or REM sleep are less likely to be reached. Third, in sleep deprivation protocols, participants must remain awake either for the full night following initial learning of a motor skill [total sleep deprivation, e.g., (Maquet et al., 2003), see Figure 12C], or for only a part of the night [partial sleep deprivation, e.g., (Plihal and Born, 1997)] as compared to control subjects who receive normal sleep during this period. In total sleep deprivation protocols, participants are then typically retested 2-3 days after initial training, thus allowing nights of recovery for those who were sleep deprived. As the retest is usually at the same time of day as initial training, this design avoids time of day effects, but does necessitate that participants stay awake for more than 24 consecutive hours. Partial sleep deprivation protocols can target continuous chunks of sleep [e.g., first vs. second halves of the night as in (Plihal and Born, 1997)] or selectively target particular sleep stages [e.g., specific online disruption of SWS or REM sleep based on PSG recordings; (Genzel et al., 2009)]. This protocol presents important limitations as training and retest take place at different time of the day/night, (e.g., $11 \mathrm{pm} / 3 \mathrm{am}$ and $3 \mathrm{am} / 7 \mathrm{am}$ for early vs. late sleep groups, respectively). It is important to note that, altogether, sleep deprivation protocols present the disadvantage that the lack of sleep is paralleled by secondary (possibly confounding) effects, such as stress or alteration of hormonal release (Joo et al., 2012). Fourth, with time-variant designs [e.g., (Albouy et al., 2008); Figure I2D), the retest for each experimental group is completed after different retention interval durations, which may or may not include sleep. For example, participants may be retested 6 or 24 hours after initial training, and only the latter group experienced sleep during the retention interval. This protocol examines the influence of a full night of sleep, as compared to a non-matched wake control group, on memory consolidation. Therefore, such designs cannot differentiate the effects of time from the effects of sleep without the inclusion of additional experimental controls. It is worth mentioning that in a comprehensive metaanalyses on the influence of sleep on offline performance enhancements for explicit motor sequence tasks, Pan \& Rickard (2015) discuss these various designs and suggest the following recommendations: a) PM / AM designs should not be employed due to the confounding influence of varying testing times; 
and, b) the Nap/No Nap and time-variant designs are favorable (but see more complete discussion in Pan \& Rickard, 2015).

Last, we wish to briefly emphasize that many studies have employed a design in which all participants are retested after an interval including sleep (i.e., $24 \mathrm{~h}$ retest) and results are discussed in the context of the intervening sleep period. If information on sleep characteristics was obtained via polysomnography (see Inset 3), then researchers can relate changes in behavior to specific sleep features (Section 4). If no such information was recorded, then interpretations of these results with respect to sleep-related benefits are limited due to the lack of appropriate wake control groups (see Inset 4).

- INSERT FIGURE I2 HERE - 


\section{Inset 3: Assessment of sleep and motor-memory related sleep features}

Polysomnography (PSG), an electrophysiological technique measuring electrical activity of the brain (with electroencephalography (EEG)) as well as ocular and muscular activity (with electrooculography (EOG) and electromyography (EMG), respectively) is considered the gold standard for the objective assessment of sleep (Carskadon and Dement, 2017). Human sleep can be divided into 4 stages by the monitoring of these physiological activities: stage N1 (NREM 1), stage N2 (NREM 2), stage N3 (NREM 3 also used to indicate the original sleep stages 3 and 4 (Rechtschaffen and Kales, 1968) and Stage R (REM). We will describe below the different sleep stages and specific sleep features that have been associated with motor memory consolidation processes.

Stage N2 sleep is characterized by the presence of K-complexes and sleep spindles. The physiology of K complexes will not be described here, as we did not find any report in the literature involving $\mathrm{K}$ complexes in motor memory consolidation processes. Spindle oscillations, consisting of $11-16 \mathrm{~Hz}(0.5-$ 3sec) waxing and waning field potentials, are electrographic landmarks of N2, but also occur during N3. Spindles are generated by thalamic and cortico-thalamic networks, superimposed with slow oscillations (Clemens et al., 2007; Mölle et al., 2002) or temporally locked to a K-complex [for reviews, see (De Gennaro and Ferrara, 2003; Sejnowski and Destexhe, 2000)]. The neurophysiological mechanisms of spindle generation have been widely delineated at the cellular level and in animal models, but less so in humans. The functions of sleep spindles are still highly speculative [for review, see (Lüthi, 2013)]; however, two major functions have been proposed: the preservation of sleep [by suppressing the thalamic transmission of external sensory stimuli to cortical regions; (Cote et al., 2000; Dang-Vu et al., 2011; Elton et al., 1997; Schabus et al., 2012) and - of high interest for the present review - the consolidation of newly acquired memories [for reviews, see (Astori et al., 2013; Rasch and Born, 2013)]. Two different types of sleep spindles have been described: slow $(<13 \mathrm{~Hz})$ and fast spindles $(>13 \mathrm{~Hz})$ that are predominant over frontal and centro-parietal regions, respectively [e.g., (Anderer et al., 2001; Jobert et al., 1992; Schabus et al., 2007)]. The sources of these slow and fast spindles are predominantly thought to be located in frontal and temporo-parietal regions [e.g., (Anderer et al., 2001; Del Felice et al., 2014)], respectively. In line with these findings, a combined EEG-fMRI study by Schabus et al. (2007) demonstrated that, whereas both spindle types share common activity patterns (e.g., thalamic activation), each type of spindle is additionally associated with activity in "type-specific" areas. Most interestingly, fast but not slow spindles are accompanied by hippocampal activity, pointing to the possibility of a specific function for the fast spindle in memory consolidation. It is worth noting that the precise definitions (i.e., frequency ranges) of slow and fast spindles are not always consistent and these analyses may often be conducted post-hoc. Thus, interpretation of results differentiating between slow and fast spindles must be made with caution.

Stage N3 is defined by slow wave activity (SWA): waves with a frequency between $0.5-2 \mathrm{~Hz}$ and a peakto-peak amplitude $>75 \mu \mathrm{V}$, measured over anterior regions. These highly synchronous oscillations indicate that large populations of neurons within the cortex fire together. SWA is homeostatically regulated (Borbély and Achermann, 1999): it increases after prolonged wakefulness or sleep deprivation. Each slow oscillation consists of a hyperpolarized "down-state" that is characterized by neuronal silence, and a depolarized "up-state" associated with high rates of cortical activity [for reviews, see (Riedner et al., 2011; Steriade et al., 1993)]. In the human EEG, slow oscillations have a spectral peak frequency of $\sim 0.8 \mathrm{~Hz}$ (Achermann and Borbély, 1997), originating from distinct cortical regions with hotspots neighboring the lateral sulci [e.g., the insula, superior temporal gyri) and travelling predominantly along 
the anterior-posterior axis of the brain; (Massimini et al., 2004; Murphy et al., 2009; Riedner et al., 2011)], often from the prefrontal cortex to the medial temporal lobe and the hippocampus (Nir et al., 2011). According to the active system consolidation hypothesis, slow oscillations orchestrate the hippocampal-to-cortical information transfer during sleep and play a critical role in systems consolidation. Slow oscillations are suggested to exert a top-down synchronizing influence on hippocampal sharp wave-ripple complexes, sleep spindles and on faster cortical beta and gamma activity (Born and Wilhelm, 2012; Diekelmann and Born, 2010; Rasch and Born, 2013). Besides this theory, slow oscillations are further argued to be critically involved in synaptic downscaling, ensuring the desaturation of synapses potentiated as a result of learning during prior wakefulness [i.e. the synaptic downscaling hypothesis; (Tononi and Cirelli, 2014, 2006, 2003). Please note that these theoretical models mainly build on findings in the declarative memory domain.

REM sleep regularly appears 60 to $90 \mathrm{~min}$ after sleep onset. Oscillatory properties within REM sleep resemble those of wakefulness and oxygen consumption in extrastriate cortical areas is even higher than during waking [see (Dang-Vu et al., 2010) for a review]. Therefore, this stage was formerly indicated as "paradoxical sleep". EEG activity during REM consists of low-voltage EEG activity, periods of alpha waves, slightly slower than during wakefulness and "sawtooth waves" $(2-6 \mathrm{~Hz})$ observed in short bursts over frontal leads or vertex. Sharp, frequent ocular potentials are evident and muscle activity measured by EMG shows atonia with only transient phasic activity (i.e., transient bursts of eye movements, myoclonic twitches and irregular vegetative activity). The physiological function of REM sleep is still largely unclear. Evidence linking REM to aspects of memory consolidation has been obtained using techniques such as statistical correlation, pharmacology, and REM sleep deprivation (Diekelmann et al., 2011; Rasch et al., 2009; Stickgold, 2005). However, whether REM has a direct role in learning and memory remains controversial and causal evidence is still missing [for review, see (Rasch and Born, 2015)].

The metrics that have been used to put sleep in relation to motor memory processes are (a) sleep stage duration and (b) measures of particular sleep features. For example, measures reflecting sleep spindles are spindle number, density, amplitude, duration, "activity" (i.e., mean spindle duration * mean spindle amplitude) but also EEG sigma power $(12-15 \mathrm{~Hz})$. Slow wave activity is commonly assessed through the analysis of EEG delta $(<4 \mathrm{~Hz})$ and slow oscillations power $(<1 \mathrm{~Hz})$. REM activity is typically assessed through density of rapid eye movements.

While PSG is considered the gold standard to record and characterize sleep stages, actigraphy can also be used as an alternative to objectively assess sleep patterns [e.g., (Appleman et al., 2016)]. Actigraphs (or actimeters) are wristwatches composed of an accelerometer recording limb movement over long periods of time (up to several weeks) and allow the estimation of activity-inactivity patterns in humans. While the estimation of sleep-wake patterns based on limb movement has some important limitations, actigraphy is a convenient, cheap and portable method that does not disturb sleep and can be very valuable in populations showing less tolerance to PSG (e.g., children and elderly). Finally, sleep quality and duration can be assessed subjectively with self-administered questionnaires like the Pittsburgh Sleep Quality Index (Buysse et al., 1989) or sleep diaries [e.g., (Carney et al., 2012)].

Note that Section 4 of the present review mainly focuses on PSG experiments but some of the studies reported in the other sections (in particular studies using PM/AM, sleep deprivation and time variant designs) made use of questionnaires and actigraphy. 


\section{Inset 4: Methodological considerations for future research}

This inset offers methodological considerations that are critical for future sleep and motor memory experiments. Although some of these may seem trivial or even obvious, our review of the literature revealed that a more detailed discussion of these particular issues is necessary. Adherence to such methodological guidelines will decrease the inconsistent results in the literature and help continue to propel research on sleep and motor memory consolidation forward. Please see Pan \& Rickard (2015) for discussion of additional methodological considerations.

Wake control group: If one of the primary research questions is whether post-learning sleep offers benefits to motor memory consolidation, it is vital to include a waking control group and to conduct appropriate between-group statistical comparisons in order to make valid conclusions about the influence of sleep. Our review of the literature revealed numerous instances when authors made conclusions about the role of post-learning sleep without having an appropriate control group. Moreover, even if an appropriate wake control group was included, there were several occasions in which the sleep and wake groups were not statistically compared. Authors would erroneously conclude that a significant between-session improvement within the sleep group and a non-significant betweensession change within the wake group was evidence for a sleep-dependent effect [see (Nieuwenhuis et al., 2011) for a discussion of this issue].

Post-training test: There is growing evidence that if a post-test is administered shortly after (e.g., 10-30 minutes) the initial learning of a sequential motor skill, a spontaneous boost in performance is observed that then disappears after longer periods (i.e., several hours) of wakefulness (Hotermans et al., 2006; Landry et al., 2016; Nettersheim et al., 2015). Critically, when such a post-test is employed, there are no additional significant performance enhancements when participants were retested after longer consolidation intervals that included sleep (Landry et al., 2016; Nettersheim et al., 2015). Although these results suggest that sleep may not enhance performance relative to the post-training test in the form of spontaneous offline gains, they do not indicate that sleep fails to contribute to motor memory consolidation processes, as there is still evidence of sleep-dependent benefit relative to wake control groups [e.g., (Nettersheim et al., 2015; Rickard et al., 2008)]. Importantly, the temporary boost in performance evident 10-30 minutes after training is thought to be related to memory processes as it predicts subsequent overnight consolidation (Hotermans et al., 2006). Thus, if the goal of a post-training test is to establish more stable and reliable estimates of post-learning performance (that are less likely influenced by confounding variables such as fatigue or decreased attention), we argue that such test should take place immediately after training (rather than after a delay of 30 minutes during which mnemonic processing has already commenced). Although the immediate post-training test is beneficial for the interpretation of within-group, across-session performance changes (i.e., performance enhancements vs. maintenance vs. deterioration), the inclusion of the wake control group and the appropriate statistical comparisons (see above) are still vital for assessing the influence of sleep on motor memory consolidation.

Interference between tasks: Future experimental designs should be restricted to the assessment of the influence of sleep on memory consolidation of a single task. Our literature review revealed numerous studies in which participants were trained and tested on both motor and declarative memory tasks, for example, before and after the sleeping episode. It has been about 10 years since Robertson and colleagues demonstrated that procedural and declarative memory systems interact during the consolidation process (Brown and Robertson, 2007) and there is also recent evidence of interference 
during subsequent recall (Gagné and Cohen, 2016). Interpretation of data following such mixed design is therefore limited and such multiple testing should be avoided in future research.

Adherence to constant sleep schedule prior to learning: As sleep quality prior to an initial learning session is thought to influence encoding of the motor skill (Appleman et al., 2016), future studies should require participants to adhere to a regular sleep schedule prior to participation. Moreover, via low-cost approaches such as actigraphy (see Inset 3), questionnaires or sleep diaries, it would be beneficial for future research to report data on the participants' sleep quantity and quality prior to the first experimental session. 


\section{Figures}

SLEEP-DEPENDENT CONSOLIDATION PROCESSES

曲 TIME-DEPENDENT CONSOLIDATION PROCESSES

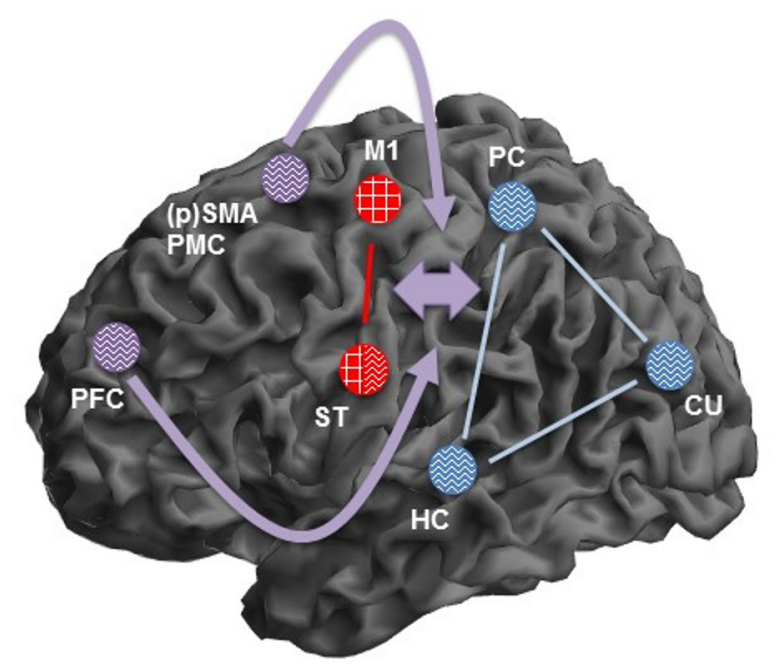

ABSTRACT REPRESENTATION

MOTORIC REPRESENTATION

BETWEEN-REPRESENTATION MODULATORS

Figure 1: Neural correlates of sleep and time-dependent motor sequence memory consolidation processes. See Section 3.1.7. for a detailed description. 
A. Motor Sequence

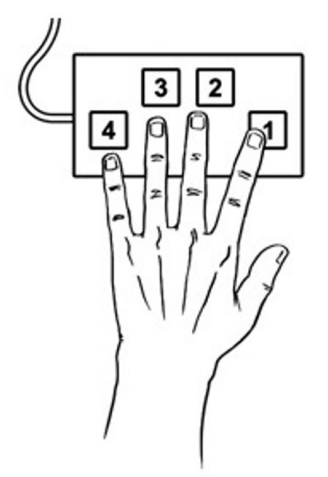

Sequence

$-41324-$
B. Motor Adaptation

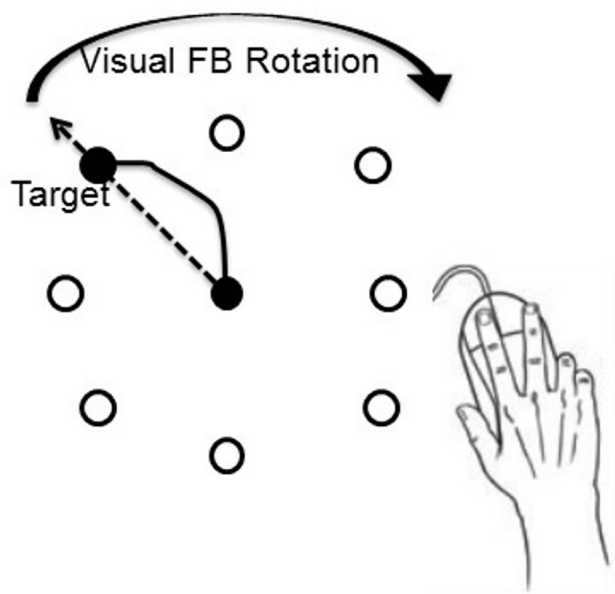

C. Continuous Tracking

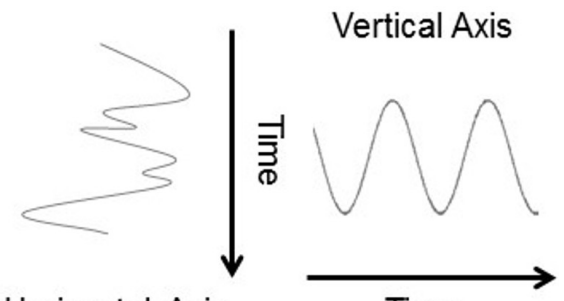

Horizontal Axis

Resulting 2D Track

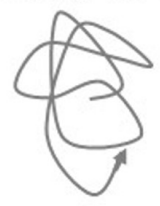

Figure 11: Exemplar depictions of variants of the three primary motor tasks used to assess the influence of sleep on motor memory consolidation processes. Inset B adapted with permission from Albouy et al., 2013d. 


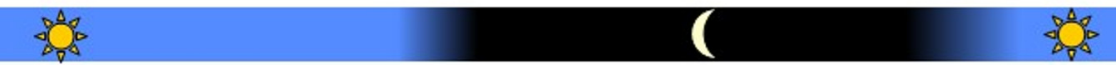

\section{A. PM / AM Design}

$\mathrm{L}$ Wake

\section{$R$}

$\mathrm{L}$

Sleep

\section{$\mathrm{R}$}

B. Nap / No Nap Design

$\mathrm{L}$ Wake $\mathrm{R}$

$\mathrm{L}$ Nap $\mathrm{R}$

C. Sleep Deprivation Design

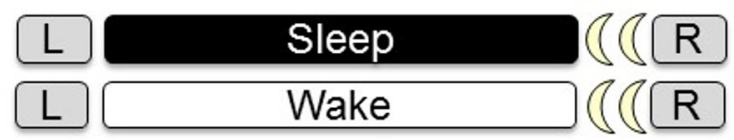

D. Time Variant Design

$\mathrm{L}$ Wake $\mathrm{R}$

$\mathrm{L}$ Wake Sleep R

Figure 12: Exemplar depictions of the four primary experimental designs used to assess the influence of sleep - as compared to wake - on motor memory consolidation processes. L=initial learning episode. $R=$ retest. 


\section{Acknowledgments}

This work was supported by the Belgian FWO Research Foundation Flanders (grant number G099516N) and the Austrian Science Fund (FWF) Project P25000. Financial support for author BRK was provided by the European Union's Horizon 2020 research and innovation programme under the Marie SkłodowskaCurie grant agreement (No 703490) and a postdoctoral fellowship from the Research Foundation Flanders (FWO; No 132635). FH was associated with and financially supported by the Doctoral College "Imaging the Mind" (FWF; W1233). 


\section{References}

Achermann, P., Borbély, A.A., 1997. Low-frequency (\&lt; $1 \mathrm{~Hz}$ ) oscillations in the human sleep electroencephalogram. Neuroscience 81, 213-22.

Adi-Japha, E., Badir, R., Dorfberger, S., Karni, A., 2014. A matter of time: rapid motor memory stabilization in childhood. Dev. Sci. 17, 424-433.

Adi-Japha, E., Karni, A., 2016. Time for considering constraints on procedural memory consolidation processes: Comment on Pan and Rickard (2015) with specific reference to developmental changes. Psychol. Bull. 142, 568-571.

Albouy, G., Fogel, S., King, B.R., Laventure, S., Benali, H., Karni, A., Carrier, J., Robertson, E., Doyon, J., 2015. Maintaining vs. enhancing motor sequence memories: Respective roles of striatal and hippocampal systems. Neuroimage 108, 423-34.

Albouy, G., Fogel, S., Pottiez, H., Nguyen, V.A., Ray, L., Lungu, O., Carrier, J., Robertson, E., Doyon, J., 2013a. Daytime sleep enhances consolidation of the spatial but not motoric representation of motor sequence memory. PLoS One 8, e52805.

Albouy, G., King, B., Schmidt, C., M, D., Dang-Vu, T., Balteau, E., Phillips, C., Degueldre, C., Orban, P., Benali, H., Peigneux, P., Luxen, A., Karni, A., Doyon, J., Maquet, P., Korman, M., 2016. Cerebral activity associated with transient sleep-facilitated reduction in motor memory vulnerability to interference. Sci. Rep. 6, 34948.

Albouy, G., King, B.R., Maquet, P., Doyon, J., 2013b. Hippocampus and striatum: Dynamics and interaction during acquisition and sleep-related motor sequence memory consolidation. Hippocampus 23, 985-1004.

Albouy, G., Ruby, P., Phillips, C., Luxen, A., Peigneux, P., Maquet, P., 2006. Implicit oculomotor sequence learning in humans: Time course of offline processing. Brain Res. 1090, 163-171.

Albouy, G., Sterpenich, V., Balteau, E., Vandewalle, G., Desseilles, M., Dang-Vu, T., Darsaud, A., Ruby, P., Luppi, P.H., Degueldre, C., Peigneux, P., Luxen, A., Maquet, P., 2008. Both the hippocampus and striatum are involved in consolidation of motor sequence memory. Neuron 58, 261-272.

Albouy, G., Sterpenich, V., Vandewalle, G., Darsaud, A., Gais, S., Rauchs, G., Desseilles, M., Boly, M., Dang-Vu, T., Balteau, E., Degueldre, C., Phillips, C., Luxen, A., Maquet, P., 2013c. Interaction between hippocampal and striatal memory systems predicts subsequent motor sequence memory 
consolidation. PLoS One 8, e59490.

Albouy, G., Sterpenich, V., Vandewalle, G., Darsaud, A., Gais, S., Rauchs, G., Desseilles, M., Boly, M., Dang-Vu, T., Balteau, E., Degueldre, C., Phillips, C., Luxen, A., Maquet, P., 2012. Neural correlates of performance variability during motor sequence acquisition. Neuroimage 60, 324-31.

Albouy, G., Vandewalle, G., Sterpenich, V., Rauchs, G., Desseilles, M., Balteau, E., Degueldre, C., Phillips, C., Luxen, A., Maquet, P., 2013d. Sleep stabilizes visuomotor adaptation memory: an functional magnetic resonance imaging study. J. Sleep Res. 22, 144-154.

Anderer, P., Klösch, G., Gruber, G., Trenker, E., Pascual-Marqui, R.D., Zeitlhofer, J., Barbanoj, M.J., Rappelsberger, P., Saletu, B., 2001. Low-resolution brain electromagnetic tomography revealed simultaneously active frontal and parietal sleep spindle sources in the human cortex. Neuroscience $103,581-92$.

Andrade, K.C., Spoormaker, V.I., Dresler, M., Wehrle, R., Holsboer, F., Sämann, P.G., Czisch, M., 2011. Sleep spindles and hippocampal functional connectivity in human NREM sleep. J. Neurosci. 31, 10331-9.

Antonenko, D., Diekelmann, S., Olsen, C., Born, J., Mölle, M., 2013. Napping to renew learning capacity: enhanced encoding after stimulation of sleep slow oscillations. Eur. J. Neurosci. 37, 1142-51.

Antony, J.W., Gobel, E.W., O’Hare, J.K., Reber, P.J., Paller, K.A., 2012. Cued memory reactivation during sleep influences skill learning. Nat. Neurosci. 15, 1114-1116.

Appleman, E.R., Albouy, G., Doyon, J., Cronin-Golomb, A., King, B.R., 2016. Sleep quality influences subsequent motor skill acquisition. Behav. Neurosci. 130, 290-297.

Ashtamker, L., Karni, A., 2013. Motor memory in childhood: Early expression of consolidation phase gains. Neurobiol. Learn. Mem. 106, 26-30.

Astori, S., Wimmer, R.D., Lüthi, A., 2013. Manipulating sleep spindles--expanding views on sleep, memory, and disease. Trends Neurosci. 36, 738-48.

Backhaus, J., Junghanns, K., 2006. Daytime naps improve procedural motor memory. Sleep Med. 7, 50812.

Backhaus, W., Braaß, H., Renné, T., Krüger, C., Gerloff, C., Hummel, F.C., 2016. Daytime sleep has no effect on the time course of motor sequence and visuomotor adaptation learning. Neurobiol. Learn. Mem. 131, 147-154.

Bapi, R.S., Doya, K., Harner, A.M., 2000. Evidence for effector independent and dependent 
representations and their differential time course of acquisition during motor sequence learning. Exp. Brain Res. 132, 149-162.

Barakat, M., Carrier, J., Debas, K., Lungu, O., Fogel, S., Vandewalle, G., Hoge, R.D., Bellec, P., Karni, A., Ungerleider, L.G., Benali, H., Doyon, J., 2013. Sleep spindles predict neural and behavioral changes in motor sequence consolidation. Hum. Brain Mapp. 34, 2918-2928.

Barakat, M., Doyon, J., Debas, K., Vandewalle, G., Morin, A., Poirier, G., Martin, N., Lafortune, M., Karni, A., Ungerleider, L.G., Benali, H., Carrier, J., 2011. Fast and slow spindle involvement in the consolidation of a new motor sequence. Behav. Brain Res. 217, 117-121.

Bergmann, T.O., Mölle, M., Schmidt, M.A., Lindner, C., Marshall, L., Born, J., Siebner, H.R., 2012. EEGguided transcranial magnetic stimulation reveals rapid shifts in motor cortical excitability during the human sleep slow oscillation. J. Neurosci. 32, 243-53.

Blischke, K., Erlacher, D., Kresin, H., Brueckner, S., Malangré, A., 2008. Benefits of sleep in motor learning - Prospects and limitations. J. Hum. Kinet. 20, 23-36.

Borbély, A.A., Achermann, P., 1999. Sleep homeostasis and models of sleep regulation. J. Biol. Rhythms 14, 557-68.

Borich, M.R., Kimberley, T.J., 2011. Both sleep and wakefulness support consolidation of continuous, goal-directed, visuomotor skill. Exp. Brain Res. 214, 619-630.

Born, J., Rasch, B., Gais, S., 2006. Sleep to remember. Neurosci. 12, 410-24.

Born, J., Wilhelm, I., 2012. System consolidation of memory during sleep. Psychol. Res. 76, 192-203.

Borragán, G., Urbain, C., Schmitz, R., Mary, A., Peigneux, P., 2015. Sleep and memory consolidation: Motor performance and proactive interference effects in sequence learning. Brain Cogn. 95, 54-61.

Bottary, R., Sonni, A., Wright, D., Spencer, R.M.C., 2016. Insufficient chunk concatenation may underlie changes in sleep-dependent consolidation of motor sequence learning in older adults. Learn. Mem. 23, 455-459.

Brashers-Krug, T., Shadmehr, R., Bizzi, E., 1996. Consolidation in human motor memory. Nature 382, $252-255$.

Brawn, T.P., Fenn, K.M., Nusbaum, H.C., Margoliash, D., 2008. Consolidation of sensorimotor learning during sleep. Learn. Mem. 15, 815-9.

Brown, R.M., Robertson, E.M., 2007. Off-line processing: reciprocal interactions between declarative and procedural memories. J. Neurosci. 27, 10468-75. 
Brown, R.M., Robertson, E.M., Press, D.Z., 2009. Sequence skill acquisition and off-line learning in normal aging. PLoS One 4, e6683.

Buchegger, J., Fritsch, R., Meier-Koll, A., Riehle, H., 1991. Does trampolining and anaerobic physical fitness affect sleep? Percept. Mot. Skills 73, 243-52.

Buysse, D.J., Reynolds, C.F., Monk, T.H., Berman, S.R., Kupfer, D.J., 1989. The Pittsburgh Sleep Quality Index: a new instrument for psychiatric practice and research. Psychiatry Res. 28, 193-213.

Cai, D.J., Rickard, T.C., 2009. Reconsidering the role of sleep for motor memory. Behav. Neurosci. 123, 1153-1157.

Caithness, G., Osu, R., Bays, P., Chase, H., Klassen, J., Kawato, M., Wolpert, D.M., Flanagan, J.R., 2004. Failure to consolidate the consolidation theory of learning for sensorimotor adaptation tasks. J. Neurosci. 24, 8662-8671.

Cajochen, C., Knoblauch, V., Wirz-Justice, A., Kräuchi, K., Graw, P., Wallach, D., 2004. Circadian modulation of sequence learning under high and low sleep pressure conditions. Behav. Brain Res. $151,167-76$.

Caporro, M., Haneef, Z., Yeh, H.J., Lenartowicz, A., Buttinelli, C., Parvizi, J., Stern, J.M., 2012. Functional MRI of sleep spindles and K-complexes. Clin. Neurophysiol. 123, 303-9.

Carlson, N., 2004. Physiologische Psychologie, 6th ed. Pearson Studium, Munchen.

Carney, C.E., Buysse, D.J., Ancoli-Israel, S., Edinger, J.D., Krystal, A.D., Lichstein, K.L., Morin, C.M., 2012. The consensus sleep diary: standardizing prospective sleep self-monitoring. Sleep 35, 287-302.

Carskadon, M.A., Dement, W.C., 2017. Normal human sleep: an overview, in: Kryger, M.H., Roth, T., Dement, W.C. (Eds.), Principles and Practice of Sleep Medicine. Elsevier, Philadelphia, PA, pp. 1524.

Carskadon, M.A., Herz, R.S., 2004. Minimal olfactory perception during sleep: why odor alarms will not work for humans. Sleep 27, 402-5.

Censor, N., Buch, E.R., Nader, K., Cohen, L.G., 2016. Altered human memory modification in the presence of normal consolidation. Cereb. Cortex 26, 3828-37. doi:180

Censor, N., Dayan, E., Cohen, L.G., 2014. Cortico-subcortical neuronal circuitry associated with reconsolidation of human procedural memories. Cortex 58, 281-8.

Chein, J.M., Schneider, W., 2005. Neuroimaging studies of practice-related change: fMRI and metaanalytic evidence of a domain-general control network for learning. Cogn. Brain Res. 25, 607-23. 
Chun, M.M., Phelps, E.A., 1999. Memory deficits for implicit contextual information in amnesic subjects with hippocampal damage. Nat. Neurosci. 2, 844-847.

Clemens, Z., Mölle, M., Eross, L., Barsi, P., Halász, P., Born, J., 2007. Temporal coupling of parahippocampal ripples, sleep spindles and slow oscillations in humans. Brain 130, 2868-78.

Clemens, Z., Mölle, M., Eross, L., Jakus, R., Rásonyi, G., Halász, P., Born, J., 2011. Fine-tuned coupling between human parahippocampal ripples and sleep spindles. Eur. J. Neurosci. 33, 511-20.

Cohen, D.A., Pascual-Leone, A., Press, D.Z., Robertson, E.M., 2005. Off-line learning of motor skill memory: a double dissociation of goal and movement. Proc. Natl. Acad. Sci. U. S. A. 102, 18237-41.

Cohen, D.A., Robertson, E.M., 2007. Motor sequence consolidation: constrained by critical time windows or competing components. Exp. Brain Res. 177, 440-446.

Cote, K.A., Epps, T.M., Campbell, K.B., 2000. The role of the spindle in human information processing of high-intensity stimuli during sleep. J. Sleep Res. 9, 19-26.

Cousins, J.N., El-Deredy, W., Parkes, L.M., Hennies, N., Lewis, P.A., 2016. Cued reactivation of motor learning during sleep leads to overnight changes in functional brain activity and connectivity. PLoS Biol. 14, e1002451.

Cousins, J.N., El-Deredy, W., Parkes, L.M., Hennies, N., Lewis, P.A., 2014. Cued memory reactivation during slow-wave sleep promotes explicit knowledge of a motor sequence. J. Neurosci. 34, 1587015876.

Cunningham, H.A., Welch, R.B., 1994. Multiple concurrent visual-motor mappings: implications for models of adaptation. J. Exp. Psychol. Hum. Percept. Perform. 20, 987-999.

Dan, E., King, B.R., Doyon, J., Chan, P., 2015. Motor sequence learning and consolidation in unilateral de novo patients with Parkinson's disease. PLoS One 10, e0134291.

Dang-Vu, T.T., Bonjean, M., Schabus, M., Boly, M., Darsaud, A., Desseilles, M., Degueldre, C., Balteau, E., Phillips, C., Luxen, A., Sejnowski, T.J., Maquet, P., 2011. Interplay between spontaneous and induced brain activity during human non-rapid eye movement sleep. Proc. Natl. Acad. Sci. U. S. A. $108,15438-43$.

Dang-Vu, T.T., Schabus, M., Desseilles, M., Sterpenich, V., Bonjean, M., Maquet, P., 2010. Functional neuroimaging insights into the physiology of human sleep. Sleep 33, 1589-603.

Datta, S., Patterson, E., 2003. Activation of phasic pontine wave ( $p$-wave): A mechanism of learning and memory processing, in: Maquet, P., Smith, C., Stickgold, R. (Eds.), Sleep and Brain Plasticity. Oxford 
University Press, Oxford, pp. 135-156.

Dayan, E., Laor-Maayany, R., Censor, N., 2016. Reward disrupts reactivated human skill memory. Sci. Rep. 6, 28270.

de Beukelaar, T.T., Woolley, D.G., Alaerts, K., Swinnen, S.P., Wenderoth, N., 2016. Reconsolidation of motor memories Is a time-dependent process. Front. Hum. Neurosci. 10, 408.

de Beukelaar, T.T., Woolley, D.G., Wenderoth, N., 2014. Gone for 60 seconds: Reactivation length determines motor memory degradation during reconsolidation. Cortex 59, 138-145.

De Gennaro, L., Ferrara, M., 2003. Sleep spindles: an overview. Sleep Med. Rev. 7, 423-40.

De Lucia, M., Constantinescu, I., Sterpenich, V., Pourtois, G., Seeck, M., Schwartz, S., 2011. Decoding sequence learning from single-trial intracranial EEG in humans. PLoS One 6, e28630.

Debarnot, U., Abichou, K., Kalenzaga, S., Sperduti, M., Piolino, P., 2015. Variable motor imagery training induces sleep memory consolidation and transfer improvements. Neurobiol. Learn. Mem. 119, 8592.

Debarnot, U., Castellani, E., Guillot, A., 2012. Selective delayed gains following motor imagery of complex movements. Arch. Ital. Biol. 150, 238-250.

Debarnot, U., Castellani, E., Valenza, G., Sebastiani, L., Guillot, A., 2011. Daytime naps improve motor imagery learning. Cogn. Affect. Behav. Neurosci. 11, 541-50.

Debarnot, U., Creveaux, T., Collet, C., Doyon, J., Guillot, A., 2009. Sleep contribution to motor memory consolidation: a motor imagery study. Sleep 32, 1559-1565.

Debas, K., Carrier, J., Barakat, M., Marrelec, G., Bellec, P., Hadj Tahar, A., Karni, A., Ungerleider, L.G., Benali, H., Doyon, J., 2014. Off-line consolidation of motor sequence learning results in greater integration within a cortico-striatal functional network. Neuroimage 99, 50-8.

Debas, K., Carrier, J., Orban, P., Barakat, M., Lungu, O., Vandewalle, G., Tahar, A.H., Bellec, P., Karni, A., Ungerleider, L.G., Benali, H., Doyon, J., 2010. Brain plasticity related to the consolidation of motor sequence learning and motor adaptation. Proc. Natl. Acad. Sci. U. S. A. 107, 17839-17844.

Del Felice, A., Arcaro, C., Storti, S.F., Fiaschi, A., Manganotti, P., 2014. Electrical source imaging of sleep spindles. Clin. EEG Neurosci. 45, 184-92.

Desrochers, P.C., Kurdziel, L.B.F., Spencer, R.M.C., 2016. Delayed benefit of naps on motor learning in preschool children. Exp. Brain Res. 234, 763-72.

Diekelmann, S., Born, J., 2010. The memory function of sleep. Nat. Rev. Neurosci. 11, 114-26. 
Diekelmann, S., Born, J., Rasch, B., 2016. Increasing explicit sequence knowledge by odor cueing during sleep in men but not women. Front. Behav. Neurosci. 10, 74.

Diekelmann, S., Büchel, C., Born, J., Rasch, B., 2011. Labile or stable: opposing consequences for memory when reactivated during waking and sleep. Nat. Neurosci. 14, 381-6.

Diekelmann, S., Wilhelm, I., Born, J., 2009. The whats and whens of sleep-dependent memory consolidation. Sleep Med. Rev. 13, 309-21.

Donchin, O., Sawaki, L., Madupu, G., Cohen, L.G., Shadmehr, R., 2002. Mechanisms influencing acquisition and recall of motor memories. J. Neurophysiol. 88, 2114-2123.

Doyon, J., Bellec, P., Amsel, R., Penhune, V., Monchi, O., Carrier, J., Lehericy, S., Benali, H., $2009 a$. Contributions of the basal ganglia and functionally related brain structures to motor learning. Behav. Brain Res. 199, 61-75.

Doyon, J., Korman, M., Morin, A., Dostie, V., Tahar, A., Benali, H., Karni, A., Ungerleider, L., Carrier, J., 2009b. Contribution of night and day sleep vs. simple passage of time to the consolidation of motor sequence and visuomotor adaptation learning. Exp. Brain Res. 195, 15-26.

Elton, M., Winter, O., Heslenfeld, D., Loewy, D., Campbell, K., Kok, A., 1997. Event-related potentials to tones in the absence and presence of sleep spindles. J. Sleep Res. 6, 78-83.

Ergorul, C., Eichenbaum, H., 2006. Essential role of the hippocampal formation in rapid learning of higher-order sequential associations. J. Neurosci. 26, 4111-7.

Ertelt, D., Witt, K., Reetz, K., Frank, W., Junghanns, K., Backhaus, J., Tadic, V., Pellicano, A., Born, J., Binkofski, F., 2012. Skill memory escaping from distraction by sleep-Evidence from dual-task performance. PLoS One 7, e50983.

Fernández-Seara, M.A., Aznárez-Sanado, M., Mengual, E., Loayza, F.R., Pastor, M.A., 2009. Continuous performance of a novel motor sequence leads to highly correlated striatal and hippocampal perfusion increases. Neuroimage 47, 1797-808.

Fischer, S., Born, J., 2009. Anticipated reward enhances offline learning during sleep. J. Exp. Psychol. Learn. Mem. Cogn. 35, 1586-1593.

Fischer, S., Drosopoulos, S., Tsen, J., Born, J., 2006. Implicit lenaring - explicit knowing: A role for sleep in memory system interaction. J. Cogn. Neurosci. 18, 311-319.

Fischer, S., Hallschmid, M., Elsner, A.L., Born, J., 2002. Sleep forms memory for finger skills. Proc. Natl. Acad. Sci. 99, 11987-91. 
Fischer, S., Nitschke, M.F., Melchert, U.H., Erdmann, C., Born, J., 2005. Motor memory consolidation in sleep shapes more effective neuronal representations. J. Neurosci. 25, 11248-55.

Fletcher, P.C., Zafiris, O., Frith, C.D., Honey, R.A.E., Corlett, P.R., Zilles, K., Fink, G.R., 2005. On the benefits of not trying: Brain activity and connectivity reflecting the interactions of explicit and implicit sequence learning. Cereb. Cortex 15, 1002-1015.

Fogel, S.M., Albouy, G., Vien, C., Popovicci, R., King, B.R., Hoge, R., Jbabdi, S., Benali, H., Karni, A., Maquet, P., Carrier, J., Doyon, J., 2014. fMRI and sleep correlates of the age-related impairment in motor memory consolidation. Hum. Brain Mapp. 35, 3625-3645.

Fogel, S.M., Smith, C.T., 2011. The function of the sleep spindle: a physiological index of intelligence and a mechanism for sleep-dependent memory consolidation. Neurosci. Biobehav. Rev. 35, 1154-65.

Fogel, S.M., Smith, C.T., 2006. Learning-dependent changes in sleep spindles and Stage 2 sleep. J. Sleep Res. 15, 250-5.

Fogel, S.M., Smith, C.T., Cote, K.A., 2007. Dissociable learning-dependent changes in REM and non-REM sleep in declarative and procedural memory systems. Behav. Brain Res. 180, 48-61.

Frankland, P.W., Bontempi, B., 2005. The organization of recent and remote memories. Nat. Rev. Neurosci. 6, 119-130.

Frey, U., Morris, R.G.M., 1997. Synaptic tagging and long-term potentiation. Nature 385, 533-536.

Gagné, M.-H., Cohen, H., 2016. Interference effects between memory systems in the acquisition of a skill. Exp. Brain Res. 234, 2883-91.

Gais, S., Köster, S., Sprenger, A., Bethke, J., Heide, W., Kimmig, H., 2008. Sleep is required for improving reaction times after training on a procedural visuo-motor task. Neurobiol. Learn. Mem. 90, 610615.

Genzel, L., Bäurle, A., Potyka, A., Wehrle, R., Adamczyk, M., Friess, E., Steiger, A., Dresler, M., 2015. Diminished nap effects on memory consolidation are seen under oral contraceptive use. Neuropsychobiology 70, 253-261.

Genzel, L., Dresler, M., Wehrle, R., Grözinger, M., Steiger, A., 2009. Slow wave sleep and REM sleep awakenings do not affect sleep dependent memory consolidation. Sleep 32, 302-10.

Genzel, L., Kiefer, T., Renner, L., Wehrle, R., Kluge, M., Grözinger, M., Steiger, A., Dresler, M., 2012a. Sex and modulatory menstrual cycle effects on sleep related memory consolidation. Psychoneuroendocrinology 37, 987-998. 
Genzel, L., Quack, A., Jäger, E., Konrad, B., Steiger, A., Dresler, M., 2012b. Complex motor sequence skills profit from sleep. Neuropsychobiology 66, 237-243.

Gheysen, F., Van Opstal, F., Roggeman, C., Van Waelvelde, H., Fias, W., 2010. Hippocampal contribution to early and later stages of implicit motor sequence learning. Exp. Brain Res. 202, 795-807.

Goedert, K.M., Willingham, D.B., 2002. Patterns of interference in sequence learning and prism adaptation inconsistent with the consolidation hypothesis. Learn. Mem. 9, 279-292.

Grafton, S.T., Hazeltine, E., Ivry, R.B., 1998. Abstract and effector-specific representations of motor sequences identified with PET. J. Neurosci. 18, 9420-8.

Gregory, M.D., Agam, Y., Selvadurai, C., Nagy, A., Vangel, M., Tucker, M., Robertson, E.M., Stickgold, R., Manoach, D.S., 2014. Resting state connectivity immediately following learning correlates with subsequent sleep-dependent enhancement of motor task performance. Neuroimage 102, 666-673.

Gudberg, C., Wulff, K., Johansen-Berg, H., 2015. Sleep-dependent motor memory consolidation in older adults depends on task demands. Neurobiol. Aging 36, 1409-1416.

Guerrien, A., Dujardin, K., Mandai, O., Sockeel, P., Leconte, P., 1989. Enhancement of memory by auditory stimulation during postlearning REM sleep in humans. Physiol. Behav. 45, 947-50.

Hallgató, E., Győri-Dani, D., Pekár, J., Janacsek, K., Nemeth, D., 2012. The differential consolidation of perceptual and motor learning in skill acquisition. Cortex 49, 1073-1081.

Hardwicke, T.E., Taqi, M., Shanks, D.R., 2016. Postretrieval new learning does not reliably induce human memory updating via reconsolidation. Proc. Natl. Acad. Sci. 113, 5206-5211.

Haxby, J. V, Grady, C.L., Horwitz, B., Ungerleider, L.G., Mishkin, M., Carson, R.E., Herscovitch, P., Schapiro, M.B., Rapoport, S.I., 1991. Dissociation of object and spatial visual processing pathways in human extrastriate cortex. Proc. Natl. Acad. Sci. U. S. A. 88, 1621-5.

Herrmann, C.S., Rach, S., Neuling, T., Strüber, D., 2013. Transcranial alternating current stimulation: a review of the underlying mechanisms and modulation of cognitive processes. Front. Hum. Neurosci. 7, 279.

Hikosaka, O., Nakahara, H., Rand, M.K., Sakai, K., Lu, X., Nakamura, K., Miyachi, S., Doya, K., 1999. Parallel neural networks for learning sequential procedures. Trends Neurosci. 22, 464-471.

Hikosaka, O., Nakamura, K., Sakai, K., Nakahara, H., 2002. Central mechanisms of motor skill learning. Curr. Opin. Neurobiol. 12, 217-222.

Hoedlmoser, K., Birklbauer, J., Schabus, M., Eibenberger, P., Rigler, S., Mueller, E., 2015. The impact of 
diurnal sleep on the consolidation of a complex gross motor adaptation task. J. Sleep Res. 24, 1009.

Holz, J., Piosczyk, H., Landmann, N., Feige, B., Spiegelhalder, K., Riemann, D., Nissen, C., Voderholzer, U., 2012. The timing of learning before night-time sleep differentially affects declarative and procedural long-term memory consolidation in adolescents. PLoS One 7, e40963.

Hotermans, C., Peigneux, P., de Noordhout, A.M., Moonen, G., Maquet, P., 2008. Repetitive transcranial magnetic stimulation over the primary motor cortex disrupts early boost but not delayed gains in performance in motor sequence learning. Eur. J. Neurosci. 28, 1216-21.

Hotermans, C., Peigneux, P., Maertens de Noordhout, A., Moonen, G., Maquet, P., 2006. Early boost and slow consolidation in motor skill learning. Learn. Mem. 13, 580-583.

Huber, R., Ghilardi, M.F., Massimini, M., Tononi, G., 2004. Local sleep and learning. Nature 430, 78-81.

Javadi, A.H., Walsh, V., Lewis, P.A., 2011. Offline consolidation of procedural skill learning is enhanced by negative emotional content. Exp. Brain Res. 208, 507-17.

Jobert, M., Poiseau, E., Jähnig, P., Schulz, H., Kubicki, S., 1992. Topographical analysis of sleep spindle activity. Neuropsychobiology 26, 210-7.

Joo, E.Y., Yoon, C.W., Koo, D.L., Kim, D., Hong, S.B., 2012. Adverse effects of 24 hours of sleep deprivation on cognition and stress hormones. J. Clin. Neurol. 8, 146-50.

Kalm, K., Davis, M.H., Norris, D., 2013. Individual sequence representations in the medial temporal lobe. J. Cogn. Neurosci. 25, 1111-21.

Karni, A., Meyer, G., Jezzard, P., Adams, M.M., Turner, R., Ungerleider, L.G., 1995. Functional MRI evidence for adult motor cortex plasticity during motor skill learning. Nature 377, 155-158.

Karni, A., Meyer, G., Rey-Hipolito, C., Jezzard, P., Adams, M.M., Turner, R., Ungerleider, L.G., 1998. The acquisition of skilled motor performance: fast and slow experience-driven changes in primary motor cortex. Proc. Natl. Acad. Sci. U. S. A. 95, 861-8.

Karni, A., Tanne, D., Rubenstein, B.S., Askenasy, J.J., Sagi, D., 1994. Dependence on REM sleep of overnight improvement of a perceptual skill. Science (80-. ). 265, 679-82.

Kempler, L., Richmond, J.L., 2012. Effect of sleep on gross motor memory. Memory 20, 907-14.

King, B., Saucier, P., Albouy, G., Fogel, S., Rumpf, J., Klann, J., Buccino, G., Binkofski, F., Classen, J., Karni, A., Doyon, J., 2016. Cerebral activation during initial motor learning forecasts subsequent sleepfacilitated memory consolidation in older adults. Cereb. Cortex. 
King, B.R., Fogel, S.M., Albouy, G., Doyon, J., 2013. Neural correlates of the age-related changes in motor sequence learning and motor adaptation in older adults. Front. Hum. Neurosci. 7, 142.

Korman, M., Doyon, J., Doljansky, J., Carrier, J., Dagan, Y., Karni, A., 2007. Daytime sleep condenses the time course of motor memory consolidation. Nat. Neurosci. 10, 1206-1213.

Korman, M., Raz, N., Flash, T., Karni, A., 2003. Multiple shifts in the representation of a motor sequence during the acquisition of skilled performance. Proc. Natl. Acad. Sci. 100, 12492-12497.

Krakauer, J.W., Ghez, C., Ghilardi, M.F., 2005. Adaptation to visuomotor transformations: consolidation, interference, and forgetting. J. Neurosci. 25, 473-478.

Kudrimoti, H.S., Barnes, C.A., McNaughton, B.L., 1999. Reactivation of hippocampal cell assemblies: effects of behavioral state, experience, and EEG dynamics. J. Neurosci. 19, 4090-101.

Kumaran, D., Maguire, E.A., 2006. The dynamics of hippocampal activation during encoding of overlapping sequences. Neuron 49, 617-629.

Kuriyama, K., Stickgold, R., Walker, M.P., 2004. Sleep-dependent learning and motor-skill complexity. Learn. Mem. 11, 705-13.

Kvint, S., Bassiri-Tehrani, B., Pruski, A., Nia, J., Nemet, I., Lopresti, M., Moisello, C., Perfetti, B., Tononi, G., Ghilardi, M.F., 2011. Acquisition and retention of motor sequences: the effects of time of the day and sleep. Arch. Ital. Biol. 149, 303-312.

Landolt, H.P., Borbely, A.A., 2001. Age-dependent changes in sleep EEG topography. Clin. Neurophysiol. $112,369-377$.

Landry, S., Anderson, C., Conduit, R., 2016. The effects of sleep, wake activity and time-on-task on offline motor sequence learning. Neurobiol. Learn. Mem. 127, 56-63.

Landsness, E.C., Crupi, D., Hulse, B.K., Peterson, M.J., Huber, R., Ansari, H., Coen, M., Cirelli, C., Benca, R.M., Ghilardi, M.F., 2009. Sleep-dependent improvement in visuomotor learning: a causal role for slow waves. Sleep 32, 1273-1284.

Lansink, C.S., Goltstein, P.M., Lankelma, J. V., Joosten, R.N.J.M.A., McNaughton, B.L., Pennartz, C.M.A., 2008. Preferential reactivation of motivationally relevant information in the ventral striatum. J. Neurosci. 28, 6372-6382.

Lansink, C.S., Goltstein, P.M., Lankelma, J. V., McNaughton, B.L., Pennartz, C.M.A., 2009. Hippocampus leads ventral striatum in replay of place-reward information. PLoS Biol. 7, e1000173.

Laureys, S., Peigneux, P., Phillips, C., Fuchs, S., Degueldre, C., Aerts, J., Del Fiore, G., Petiau, C., Luxen, A., 
van der Linden, M., Cleeremans, A., Smith, C., Maquet, P., 2001. Experience-dependent changes in cerebral functional connectivity during human rapid eye movement sleep. Neuroscience 105, 5215.

Laventure, S., Fogel, S., Lungu, O., Albouy, G., Sévigny-Dupont, P., Vien, C., Sayour, C., Carrier, J., Benali, H., Doyon, J., 2016. NREM2 and sleep spindles are instrumental to the consolidation of motor sequence memories. PLoS Biol. 14, e1002429.

Lewis, P.A., Couch, T.J., Walker, M.P., 2011. Keeping time in your sleep: Overnight consolidation of temporal rhythm. Neuropsychologia 49, 115-123.

Lustenberger, C., Boyle, M.R., Alagapan, S., Mellin, J.M., Vaughn, B. V, Fröhlich, F., 2016. Feedbackcontrolled transcranial Aaternating current stimulation reveals a functional role of sleep spindles in motor memory consolidation. Curr. Biol. 26, 2127-36.

Lüthi, A., 2013. Sleep Spindles: Where They Come From, What They Do. Neurosci. 20, 243-256.

Malangré, A., Leinen, P., Blischke, K., 2014. Sleep-related offline learning in a complex arm movement sequence. J. Hum. Kinet. 40, 7-20.

Manns, J.R., Howard, M.W., Eichenbaum, H., 2007. Gradual changes in hippocampal activity support remembering the order of events. Neuron 56, 530-40.

Mantua, J., Baran, B., Spencer, R.M.C., 2015. Sleep benefits consolidation of visuo-motor adaptation learning in older adults. Exp. Brain Res. 234, 587-595.

Maquet, P., 2000. Sleep on it! Nat. Neurosci. 3, 1235-1236.

Maquet, P., Laureys, S., Peigneux, P., Fuchs, S., Petiau, C., Phillips, C., Aerts, J., Del Fiore, G., Degueldre, C., Meulemans, T., Luxen, A., Franck, G., Van Der Linden, M., Smith, C., Cleeremans, A., 2000. Experience-dependent changes in cerebral activation during human REM sleep. Nat. Neurosci. 3, 831-836.

Maquet, P., Schwartz, S., Passingham, R., Frith, C., 2003. Sleep-related consolidation of a visuomotor skill: brain mechanisms as assessed by functional magnetic resonance imaging. J. Neurosci. 23, $1432-40$.

Marcus, C.L., Omlin, K.J., Basinki, D.J., Bailey, S.L., Rachal, A.B., Von Pechmann, W.S., Keens, T.G., Ward, S.L.D., 1992. Normal polysomnographic values for children and adolescents. Am. Rev. Respir. Dis. $146,1235-1239$.

Marshall, L., Helgadóttir, H., Mölle, M., Born, J., 2006. Boosting slow oscillations during sleep potentiates 
memory. Nature 444, 610-3.

Marshall, L., Kirov, R., Brade, J., Mölle, M., Born, J., 2011. Transcranial electrical currents to probe EEG brain rhythms and memory consolidation during sleep in humans. PLoS One 6, e16905.

Marshall, L., Mölle, M., Hallschmid, M., Born, J., 2004. Transcranial direct current stimulation during sleep improves declarative memory. J. Neurosci. 24, 9985-92.

Massimini, M., Huber, R., Ferrarelli, F., Hill, S., Tononi, G., 2004. The sleep slow oscillation as a traveling wave. J. Neurosci. 24, 6862-70.

Massimini, M., Rosanova, M., Mariotti, M., 2003. EEG slow (approximately $1 \mathrm{~Hz}$ ) waves are associated with nonstationarity of thalamo-cortical sensory processing in the sleeping human. J. Neurophysiol. 89, 1205-13.

McClain, I.J., Lustenberger, C., Achermann, P., Lassonde, J.M., Kurth, S., LeBourgeois, M.K., 2016. Developmental changes in sleep spindle characteristics and sigma power across early childhood. Neural Plast. 2016, 3670951.

McClelland, J.L., McNaughton, B.L., O’Reilly, R.C., 1995. Why there are complementary learning systems in the hippocampus and neocortex: insights from the successes and failures of connectionist models of learning and memory. Psychol. Rev. 102, 419-457.

Meier, B., Cock, J., 2014. Offline consolidation in implicit sequence learning. Cortex 57, 156-166.

Miller, E.K., Cohen, J.D., 2001. An integrative theory of prefrontal cortex function. Annu. Rev. Neurosci. 24, 167-202.

Milner, C.E., Fogel, S.M., Cote, K.A., 2006. Habitual napping moderates motor performance improvements following a short daytime nap. Biol. Psychol. 73, 141-56.

Mölle, M., Marshall, L., Gais, S., Born, J., 2002. Grouping of spindle activity during slow oscillations in human non-rapid eye movement sleep. J. Neurosci. 22, 10941-7.

Moncada, D., Ballarini, F., Viola, H., 2015. Behavioral tagging: A translation of the synaptic tagging and capture hypothesis. Neural Plast. 2015, 1-21.

Montgomery-Downs, H.E., O’Brien, L.M., Gulliver, T.E., Gozal, D., 2006. Polysomnographic characteristics in normal preschool and early school-aged children. Pediatrics 117, 741-753.

Morin, A., Doyon, J., Dostie, V., Barakat, M., Tahar, A.H., Korman, M., Benali, H., Karni, A., Ungerleider, L.G., Carrier, J., 2008. Motor sequence learning increases sleep spindles and fast frequencies in post-training sleep. Sleep 31, 1149-1156. 
Morita, Y., Ogawa, K., Uchida, S., 2016. Napping after complex motor learning enhances juggling performance. Sleep Sci. 9, 112-116.

Morita, Y., Ogawa, K., Uchida, S., 2012. The effect of a daytime 2-hour nap on complex motor skill learning. Sleep Biol. Rhythms 10, 302-309.

Moroni, F., Nobili, L., Curcio, G., De Carli, F., Tempesta, D., Marzano, C., De Gennaro, L., Mai, R., Francione, S., Lo Russo, G., Ferrara, M., 2008. Procedural learning and sleep hippocampal low frequencies in humans. Neuroimage 42, 911-918.

Murphy, M., Riedner, B.A., Huber, R., Massimini, M., Ferrarelli, F., Tononi, G., 2009. Source modeling sleep slow waves. Proc. Natl. Acad. Sci. U. S. A. 106, 1608-13.

Nemeth, D., Hallgató, E., Janacsek, K., Sándor, T., Londe, Z., 2009. Perceptual and motor factors of implicit skill learning. Neuroreport 20, 1654-1658.

Nemeth, D., Janacsek, K., 2010. The dynamics of implicit skill consolidation in young and elderly adults. Journals Gerontol. Ser. B Psychol. Sci. Soc. Sci. 66, 15-22.

Nemeth, D., Janacsek, K., Londe, Z., Ullman, M.T., Howard, D. V, Howard, J.H., 2010. Sleep has no critical role in implicit motor sequence learning in young and old adults. Exp. Brain Res. 201, 351-8.

Nettersheim, A., Hallschmid, M., Born, J., Diekelmann, S., 2015. The role of sleep in motor sequence consolidation: Stabilization rather than enhancement. J. Neurosci. 35, 6696-6702.

Nieuwenhuis, S., Forstmann, B.U., Wagenmakers, E.-J., 2011. Erroneous analyses of interactions in neuroscience: a problem of significance. Nat. Neurosci. 14, 1105-1107.

Nir, Y., Staba, R.J., Andrillon, T., Vyazovskiy, V. V, Cirelli, C., Fried, I., Tononi, G., 2011. Regional slow waves and spindles in human sleep. Neuron 70, 153-69.

Nishida, M., Walker, M.P., 2007. Daytime naps, motor memory consolidation and regionally specific sleep spindles. PLoS One 2, e341.

Nissen, M.J., Bullemer, P., 1987. Attentional requirements of learning: Evidence from performance measures. Cogn. Psychol. 19, 1-32.

Nitsche, M.A., Jakoubkova, M., Thirugnanasambandam, N., Schmalfuss, L., Hullemann, S., Sonka, K., Paulus, W., Trenkwalder, C., Happe, S., 2010. Contribution of the premotor cortex to consolidation of motor sequence learning in humans during sleep. J. Neurophysiol. 104, 2603-14.

Nitsche, M.A., Paulus, W., 2011. Transcranial direct current stimulation--update 2011. Restor. Neurol. Neurosci. 29, 463-92. 
Ohayon, M.M., Carskadon, M.A., Guilleminault, C., Vitiello, M. V, 2004. Meta-analysis of quantitative sleep parameters from childhood to old age in healthy individuals: developing normative sleep values across the human lifespan. Sleep 27, 1255-1273.

Oudiette, D., Paller, K.A., 2013. Upgrading the sleeping brain with targeted memory reactivation. Trends Cogn. Sci. 17, 142-9.

Oudiette, D., Santostasi, G., Paller, K.A., 2013. Reinforcing rhythms in the sleeping brain with a computerized metronome. Neuron 78, 413-5.

Pace-Schott, E.F., Spencer, R.M.C., 2013. Age-related changes in consolidation of perceptual and musclebased learning of motor skills. Front. Aging Neurosci. 5, 83.

Pan, S.C., Rickard, T.C., 2015. Sleep and motor earning: Is there room for consolidation? Psychol. Bull. $141,812-34$.

Peigneux, P., Laureys, S., Fuchs, S., Collette, F., Perrin, F., Reggers, J., Phillips, C., Degueldre, C., Del Fiore, G., Aerts, J., Luxen, A., Maquet, P., 2004. Are spatial memories strengthened in the human hippocampus during slow wave sleep? Neuron 44, 535-545.

Peigneux, P., Laureys, S., Fuchs, S., Destrebecqz, A., Collette, F., Delbeuck, X., Phillips, C., Aerts, J., Del Fiore, G., Degueldre, C., Luxen, A., Cleeremans, A., Maquet, P., 2003. Learned material content and acquisition level modulate cerebral reactivation during posttraining rapid-eye-movements sleep. Neuroimage 20, 125-134.

Pennartz, C.M.A., Lee, E., Verheul, J., Lipa, P., Barnes, C.A., McNaughton, B.L., 2004. The ventral striatum in off-line processing: Ensemble reactivation during sleep and modulation by hippocampal ripples. J. Neurosci. 24, 6446-6456.

Peters, K.R., Ray, L., Smith, V., Smith, C., 2008. Changes in the density of stage 2 sleep spindles following motor learning in young and older adults. J. Sleep Res. 17, 23-33.

Peters, K.R., Smith, V., Smith, C.T., 2007. Changes in sleep architecture following motor learning depend on initial skill level. J. Cogn. Neurosci. 19, 817-29.

Plihal, W., Born, J., 1997. Effects of early and late nocturnal sleep on declarative and procedural memory. J. Cogn. Neurosci. 9, 534-547.

Rasch, B., Born, J., 2015. In search of a role of REM sleep in memory formation. Neurobiol. Learn. Mem. $122,1-3$.

Rasch, B., Born, J., 2013. About sleep's role in memory. Physiol. Rev. 93, 681-766. 
Rasch, B., Büchel, C., Gais, S., Born, J., 2007. Odor cues during slow-wave sleep prompt declarative memory consolidation. Science (80-. ). 315, 1426-9.

Rasch, B., Pommer, J., Diekelmann, S., Born, J., 2009. Pharmacological REM sleep suppression paradoxically improves rather than impairs skill memory. Nat. Neurosci. 12, 396-7.

Rechtschaffen, A., Kales, A., 1968. A manual of standardized terminology, techniques and scoring system for sleep stages of human subjects. UCLA Brain Inf. Serv. Brain Res. Inst.

Reis, J., Fischer, J.T., Prichard, G., Weiller, C., Cohen, L.G., Fritsch, B., 2015. Time- but not sleepdependent consolidation of tDCS-enhanced visuomotor skills. Cereb. Cortex 25, 109-117.

Rickard, T.C., Cai, D.J., Rieth, C.A., Jones, J., Ard, M.C., 2008. Sleep does not enhance motor sequence learning. J. Exp. Psychol. Learn. Mem. Cogn. 34, 834-42.

Rickard, T.C., Pan, S.C., 2017. Time for considering the possibility that sleep plays no unique role in motor memory consolidation: Reply to Adi-Japha and Karni (2016). Psychol. Bull. 143, 454-458.

Rieckmann, A., Fischer, H., Bäckman, L., 2010. Activation in striatum and medial temporal lobe during sequence learning in younger and older adults: relations to performance. Neuroimage 50, 1303-12.

Riedner, B.A., Hulse, B.K., Murphy, M.J., Ferrarelli, F., Tononi, G., 2011. Temporal dynamics of cortical sources underlying spontaneous and peripherally evoked slow waves. Prog. Brain Res. 193, 201-18.

Rieth, C.A., Cai, D.J., McDevitt, E.A., Mednick, S.C., 2010. The role of sleep and practice in implicit and explicit motor learning. Behav. Brain Res. 214, 470-4.

Rihm, J.S., Diekelmann, S., Born, J., Rasch, B., 2014. Reactivating memories during sleep by odors: odor specificity and associated changes in sleep oscillations. J. Cogn. Neurosci. 26, 1806-18.

Robertson, E.M., 2012. New insights in human memory interference and consolidation. Curr. Biol. 22, R66-R71.

Robertson, E.M., Pascual-Leone, A., Miall, R.C., 2004a. Current concepts in procedural consolidation. Nat. Rev. Neurosci. 5, 576-582.

Robertson, E.M., Pascual-Leone, A., Press, D.Z., 2004b. Awareness modifies the skill-learning benefits of sleep. Curr. Biol. 14, 208-12.

Robertson, E.M., Press, D.Z., Pascual-Leone, A., 2005. Off-line learning and the primary motor cortex. J. Neurosci. 25, 6372-8.

Roig, M., Ritterband-Rosenbaum, A., Lundbye-Jensen, J., Nielsen, J.B., 2014. Aging increases the susceptibility to motor memory interference and reduces off-line gains in motor skill learning. 
Neurobiol. Aging 35, 1892-900.

Rosanova, M., Ulrich, D., 2005. Pattern-specific associative long-term potentiation induced by a sleep spindle-related spike train. J. Neurosci. 25, 9398-405.

Rose, M., Haider, H., Salari, N., Büchel, C., 2011. Functional dissociation of hippocampal mechanism during implicit learning based on the domain of associations. J. Neurosci. 31, 13739-45.

Ross, R.S., Brown, T.I., Stern, C.E., 2009. The retrieval of learned sequences engages the hippocampus: Evidence from fMRI. Hippocampus 19, 790-9.

Rudoy, J.D., Voss, J.L., Westerberg, C.E., Paller, K.A., 2009. Strengthening individual memories by reactivating them during sleep. Science (80-. ). 326, 1079.

Rumpf, J.-J., Wegscheider, M., Hinselmann, K., Fricke, C., King, B.R., Weise, D., Klann, J., Binkofski, F., Buccino, G., Karni, A., Doyon, J., Classen, J., 2017. Enhancement of motor consolidation by posttraining transcranial direct current stimulation in older people. Neurobiol. Aging 49, 1-8.

Sandrini, M., Cohen, L.G., Censor, N., 2015. Modulating reconsolidation: a link to causal systems-level dynamics of human memories. Trends Cogn. Sci. 19, 475-482.

Sara, S.J., 2017. Sleep to Remember. J. Neurosci. 37.

Schabus, M., Dang-Vu, T.T., Albouy, G., Balteau, E., Boly, M., Carrier, J., Darsaud, A., Degueldre, C., Desseilles, M., Gais, S., Phillips, C., Rauchs, G., Schnakers, C., Sterpenich, V., Vandewalle, G., Luxen, A., Maquet, P., 2007. Hemodynamic cerebral correlates of sleep spindles during human non-rapid eye movement sleep. Proc. Natl. Acad. Sci. U. S. A. 104, 13164-9.

Schabus, M., Dang-Vu, T.T., Heib, D.P.J., Boly, M., Desseilles, M., Vandewalle, G., Schmidt, C., Albouy, G., Darsaud, A., Gais, S., Degueldre, C., Balteau, E., Phillips, C., Luxen, A., Maquet, P., 2012. The fate of incoming stimuli during NREM sleep is determined by spindles and the phase of the slow oscillation. Front. Neurol. 3, 40.

Schendan, H.E., Searl, M.M., Melrose, R.J., Stern, C.E., 2003. An fMRI study of the role of the medial temporal lobe in implicit and explicit sequence learning. Neuron 37, 1013-25.

Schmidt, R.A., 1975. A schema theory of discrete motor skill learning. Psychol. Rev. 82, 225-260.

Schönauer, M., Geisler, T., Gais, S., 2014. Strengthening procedural memories by reactivation in sleep. J. Cogn. Neurosci. 26, 143-153.

Schönauer, M., Grätsch, M., Gais, S., 2015. Evidence for two distinct sleep-related long-term memory consolidation processes. Cortex 63, 68-78. 
Schreiner, T., Rasch, B., 2015. Boosting vocabulary learning by verbal cueing during sleep. Cereb. Cortex 25, 4169-79.

Seeck-Hirschner, M., Baier, P.C., Sever, S., Buschbacher, A., Aldenhoff, J.B., Göder, R., 2010. Effects of daytime naps on procedural and declarative memory in patients with schizophrenia. J. Psychiatr. Res. 44, 42-7.

Sejnowski, T.J., Destexhe, A., 2000. Why do we sleep? Brain Res. 886, 208-223.

Shadmehr, R., Brashers-Krug, T., 1997. Functional stages in the formation of human long-term motor memory. J. Neurosci. 17, 409-419.

Shadmehr, R., Mussa-Ivaldi, F.A., 1994. Adaptive representation of dynamics during learning of a motor task. J. Neurosci. 14, 3208-3224.

Sheth, B.R., Janvelyan, D., Khan, M., 2008. Practice makes imperfect: Restorative effects of sleep on motor learning. PLoS One 3, e3190.

Siapas, A.G., Wilson, M.A., 1998. Coordinated interactions between hippocampal ripples and cortical spindles during slow-wave sleep. Neuron $21,1123-8$.

Siengsukon, C.F., Al-Sharman, A., 2011. Sleep promotes offline enhancement of an explicitly learned discrete but not an explicitly learned continuous task. Nat. Sci. Sleep 3, 39-46.

Smith, C., 1985. Sleep states and learning: a review of the animal literature. Neurosci. Biobehav. Rev. 9, 157-68.

Smith, C., Aubrey, J., Peters, K.R., 2004. Different roles for REM and stage 2 sleep in motor learning: A proposed model. Psychol. Belg. 44, 79-102.

Smith, C., B Aubrey, J., R Peters, K., 2004. Different roles for REM and stage 2 sleep In motor learning: A proposed model. Psychol. Belg. 44, 81-104.

Smith, C., MacNeill, C., 1994. Impaired motor memory for a pursuit rotor task following Stage 2 sleep loss in college students. J. Sleep Res. 3, 206-213.

Song, S., Cohen, L.G., 2014. Practice and sleep form different aspects of skill. Nat. Commun. 5, 3407.

Song, S., Howard, J.H., Howard, D. V, 2007. Sleep does not benefit probabilistic motor sequence learning. J. Neurosci. 27, 12475-83.

Sonni, A., Spencer, R.M.C., 2015. Sleep protects memories from interference in older adults. Neurobiol. Aging 36, 2272-81.

Spencer, R.M.C., Gouw, A.M., Ivry, R.B., 2007. Age-related decline of sleep-dependent consolidation. 
Learn. Mem. 14, 480-484.

Spencer, R.M.C., Sunm, M., Ivry, R.B., 2006. Sleep-dependent consolidation of contextual learning. Curr. Biol. 16, 1001-5.

Steele, C.J., Penhune, V.B., 2010. Specific increases within global decreases: a functional magnetic resonance imaging investigation of five days of motor sequence learning. J. Neurosci. 30, 8332-41.

Steriade, M., Nuñez, A., Amzica, F., 1993. A novel slow $(<1 \mathrm{~Hz})$ oscillation of neocortical neurons in vivo: depolarizing and hyperpolarizing components. J. Neurosci. 13, 3252-65.

Sterpenich, V., Schmidt, C., Albouy, G., Matarazzo, L., Vanhaudenhuyse, A., Boveroux, P., Degueldre, C., Leclercq, Y., Balteau, E., Collette, F., Luxen, A., Phillips, C., Maquet, P., 2014. Memory reactivation during rapid eye movement sleep promotes its generalization and integration in cortical stores. Sleep 37, 1061-75.

Stickgold, R., 2005. Sleep-dependent memory consolidation. Nature 437, 1272-1278.

Stickgold, R., James, L., Hobson, J.A., 2000. Visual discrimination learning requires sleep after training. Nat. Neurosci. 3, 1237-1238.

Tamaki, M., Huang, T.-R., Yotsumoto, Y., Hämäläinen, M., Lin, F.-H., Náñez, J.E., Watanabe, T., Sasaki, Y., 2013. Enhanced spontaneous oscillations in the supplementary motor area are associated with sleep-dependent offline learning of finger-tapping motor-sequence task. J. Neurosci. 33, 13894902.

Tamaki, M., Matsuoka, T., Nittono, H., Hori, T., 2009. Activation of fast sleep spindles at the premotor cortex and parietal areas contributes to motor learning: a study using sLORETA. Clin. Neurophysiol. $120,878-86$.

Tamaki, M., Matsuoka, T., Nittono, H., Hori, T., 2008. Fast sleep spindle (13-15 hz) activity correlates with sleep-dependent improvement in visuomotor performance. Sleep 31, 204-11.

Tononi, G., Cirelli, C., 2014. Sleep and the price of plasticity: from synaptic and cellular homeostasis to memory consolidation and integration. Neuron 81, 12-34.

Tononi, G., Cirelli, C., 2006. Sleep function and synaptic homeostasis. Sleep Med. Rev. 10, 49-62.

Tononi, G., Cirelli, C., 2003. Sleep and synaptic homeostasis: a hypothesis. Brain Res. Bull. 62, 143-50.

Tucker, M.A., Fishbein, W., 2009. The impact of sleep duration and subject intelligence on declarative and motor memory performance: how much is enough? J. Sleep Res. 18, 304-12.

Tucker, M.A., Hirota, Y., Wamsley, E.J., Lau, H., Chaklader, A., Fishbein, W., 2006. A daytime nap 
containing solely non-REM sleep enhances declarative but not procedural memory. Neurobiol. Learn. Mem. 86, 241-7.

Tucker, M.A., Nguyen, N., Stickgold, R., 2016. Experience playing a musical instrument and overnight sleep enhance performance on a sequential typing task. PLoS One 11, e0159608.

Tyvaert, L., Levan, P., Grova, C., Dubeau, F., Gotman, J., 2008. Effects of fluctuating physiological rhythms during prolonged EEG-fMRI studies. Clin. Neurophysiol. 119, 2762-74.

Urbain, C., Schmitz, R., Schmidt, C., Cleeremans, A., Van Bogaert, P., Maquet, P., Peigneux, P., 2013. Sleep-dependent neurophysiological processes in implicit sequence learning. J. Cogn. Neurosci. 25, 2003-2014.

Van Der Werf, Y.D., Van Der Helm, E., Schoonheim, M.M., Ridderikhoff, A., Van Someren, E.J.W., 2009. Learning by observation requires an early sleep window. Proc. Natl. Acad. Sci. 106, 18926-18930.

van Dongen, E. V, Takashima, A., Barth, M., Zapp, J., Schad, L.R., Paller, K.A., Fernández, G., 2012. Memory stabilization with targeted reactivation during human slow-wave sleep. Proc. Natl. Acad. Sci. U. S. A. 109, 10575-80.

Verweij, I.M., Onuki, Y., Van Someren, E.J.W., Van der Werf, Y.D., 2016. Sleep to the beat: A nap favours consolidation of timing. Behav. Neurosci. 130, 298-304.

Walker, M.P., Brakefield, T., Hobson, J.A., Stickgold, R., 2003. Dissociable stages of human memory consolidation and reconsolidation. Nature 425, 616-620.

Walker, M.P., Brakefield, T., Morgan, A., Hobson, J.A., Stickgold, R., 2002. Practice with sleep makes erfect: Sleep-dependent motor skill learning. Neuron 35, 205-211.

Walker, M.P., Stickgold, R., Alsop, D., Gaab, N., Schlaug, G., 2005. Sleep-dependent motor memory plasticity in the human brain. Neuroscience 133, 911-7.

Wamsley, E.J., Hamilton, K., Graveline, Y., Manceor, S., Parr, E., 2016. Test expectation enhances memory consolidation across both sleep and wake. PLoS One 11, e0165141.

Wilhelm, I., Diekelmann, S., Molzow, I., Ayoub, A., Mölle, M., Born, J., 2011. Sleep selectively enhances memory expected to be of future relevance. J. Neurosci. 31, 1563-9.

Wilhelm, I., Metzkow-Mészàros, M., Knapp, S., Born, J., 2012. Sleep-dependent consolidation of procedural motor memories in children and adults: the pre-sleep level of performance matters. Dev. Sci. 15, 506-515.

Wilhelm, I., Rose, M., Imhof, K.I., Rasch, B., Büchel, C., Born, J., 2013. The sleeping child outplays the 
adult's capacity to convert implicit into explicit knowledge. Nat. Neurosci. 16, 391-3.

Willingham, D.B., 1999. Implicit motor sequence learning is not purely perceptual. Mem. Cognit. 27, 56172.

Wilson, J.K., Baran, B., Pace-Schott, E.F., Ivry, R.B., Spencer, R.M.C., 2012. Sleep modulates word-pair learning but not motor sequence learning in healthy older adults. Neurobiol. Aging 33, 991-1000.

Witt, K., Margraf, N., Bieber, C., Born, J., Deuschl, G., 2010. Sleep consolidates the effector-independent representation of a motor skill. Neuroscience 171, 227-234.

Wurm, M.F., Lingnau, A., 2015. Decoding actions at different levels of abstraction. J. Neurosci. 35, 772735.

Wymbs, N.F., Bastian, A.J., Celnik, P.A., 2016. Motor skills are strengthened through reconsolidation. Curr. Biol. 26, 338-343.

Yarrow, K., Brown, P., Krakauer, J.W., 2009. Inside the brain of an elite athlete: the neural processes that support high achievement in sports. Nat. Rev. Neurosci. 10, 585-96. 